\title{
Numerical robustness of single-layer method with Fourier basis for multiple obstacle acoustic scattering in homogeneous media
}

\author{
Hélène Barucq ${ }^{1,2}$, Juliette Chabassier $^{1,2},{\text { Ha } \text { Pham }^{1,2} \text {, Sébastien Tordeux }}^{2,1}$
}

\begin{abstract}
We investigate efficient methods to simulate the multiple scattering of obstacles in homogeneous media. With a large number of small obstacles on a large domain, optimized pieces of software based on spatial discretization such as Finite Element Method (FEM) or Finite Difference lose their robustness. As an alternative, we work with an integral equation method, which uses singlelayer potentials and truncation of Fourier series to describe the approximate scattered field. In the theoretical part of the paper, we describe in detail the linear systems generated by the method for impenetrable obstacles, accompanied by a well-posedness study. For the numerical performance study, we limit ourselves to the case of circular obstacles. We first compare and validate our codes with the highly optimized FEM-based software Montjoie. Secondly, we investigate the efficiency of different solver types (direct and iterative of type GMRES) in solving the dense linear system generated by the method. We observe the robustness of direct solvers over iterative ones for closely-spaced obstacles, and that of GMRES with Lower-Upper Symmetric Gauss-Seidel and Symmetric Gauss-Seidel preconditioners for far-apart obstacles.
\end{abstract}

Keywords: Multiple scattering, small obstacle acoustic scattering, single layer methods, preconditioning in multiple scattering.

\section{Introduction}

In this paper, we study efficient numerical methods to simulate the multiple acoustic scattering by a large number of small obstacles in a two-dimensional large and homogeneous media. In the presence of obstacles, an incident wave $u^{\mathbf{I}}$ is scattered, and the total field $u^{\mathbf{T}}$ is given as a superposition of the incident field $u^{\mathbf{I}}$ and the scattered one denoted by $u$, i.e. $u^{\mathbf{T}}=u^{\mathbf{I}}+u$, see Figure 1 .

\footnotetext{
Email addresses: helene.barucq@inria.fr (Hélène Barucq), juliette.chabassier@inria.fr (Juliette Chabassier), ha.howard@inria.fr (Ha Pham), sebastien.tordeux@inria.fr (Sébastien Tordeux)

${ }^{1}$ INRIA Bordeaux Sud-Ouest Research Center. Team Project Magique-3D.

${ }^{2}$ Université de Pau et des Pays de l'Adour, France.
} 
For a time-harmonic acoustic wave $u^{\mathbf{I}}(x) e^{\mathrm{i} 2 \pi \mathrm{f} t}$ (of frequency $\mathrm{f}$ ), propagating in a homogeneous medium with wave speed $c$, the waves are required to solve the Helmholtz equation

$$
\left(-\Delta-\kappa^{2}\right) w=0
$$

in $\mathbb{R}^{2}$ for $u^{\mathbf{I}}$, and in the domain outside the obstacles, defined by $\Omega_{\text {ext }}$, for $u^{\mathbf{T}}$ and $u$. Here, wavenumber $\kappa$ is determined by the dispersion relation $\kappa=\frac{2 \pi \mathrm{f}}{c}$ and the corresponding wavelength $\lambda$ is given by $\lambda=\frac{2 \pi}{\kappa}$. The scattered field $u$ 5 also needs to satisfy the $\kappa$-outgoing condition at infinity to ensure that it does not re-enter the domain of interest.
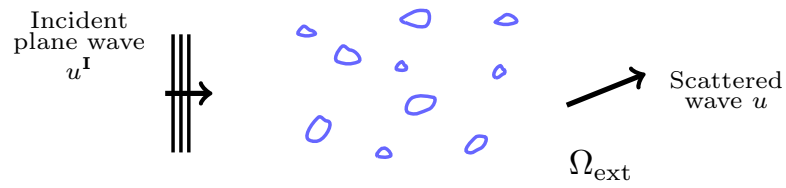

Figure 1: Scattering of a plane wave by various and non-overlapping obstacles. $\Omega_{\text {ext }}$ is the domain outside the obstacles.

We denote by $\Omega_{\text {hetero }}$ the smallest open set outside of which the medium

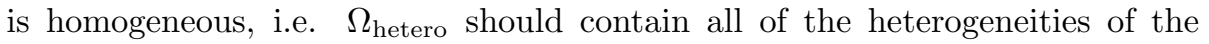
problem, in our case, the obstacles. For the approximate wave $u_{h}$, we denote by $\Omega_{\text {dist }}$ the domain of discretization ${ }^{3}$, and by $\Omega_{\text {visu }}$ the domain of visualization represented by a structured grid, $\Omega_{\mathrm{visu}} \subset \Omega_{\text {dist }}$. The sizes of the domain and the obstacles are considered relatively to the wavelength $\lambda$ of the incident wave.

In this paper, we consider configurations with one or a combination of the following factors: large $(\geq 100 \lambda) \Omega_{\text {visu }}(\mathbf{F . 1})$, with small obstacles (radius $\leq$ $15 \quad 0.3 \lambda)(\mathbf{F . 2})$, and with a great number $\left(\geq 10^{2}\right)$ of such small obstacles $(\mathbf{F . 3})$. Our focus will be more on $\mathbf{F . 2}$ and $\mathbf{F . 3}$. That the continuous problem is defined on an unbounded domain is not a major concern and can be handled efficiently with various existing methods ${ }^{4}$ for domain truncation (i.e. to render $\Omega_{\text {dist }}$ finite). The usual tools on configurations with a small number of sizable obstacles are highly optimized software based on volume discretization together with a numerical method for domain truncation. An example of these is Montjoie (montjoie. gforge.inria.fr) using spectral FEM and Perfectly Matched Layer (PML)

\footnotetext{
${ }^{3}$ This is only relevant for volume discretization methods e.g. FEM, Finite difference (FD), Finite Volume (FV), etc. For impenetrable obstacles, as in our problem, the inside of the obstacles is not meshed, i.e $\left(\Omega_{\text {hetero }} \backslash \Omega_{\text {Obs }}\right) \subset \Omega_{\text {dist }}$. Here $\Omega_{\text {Obs }}$ is the union of the interior of the obstacles.

${ }^{4}$ For a review of this subject, see [1, 2]. A more current trend is to couple (in various ways) Boundary Integral Equation (BIE) with a volume discretization method, c.f. [3, 4, 5] for a review and the references therein. BIE is efficient in handling (homogeneous) infinity since they are (volume) mesh-free and produce Ansatzs by using layer potentials which satisfy automatically the radiation condition at infinity.

${ }^{5}$ In 2D, Montjoie can handle reasonably well domains of size $\leq 100$ wavelengths. Problems beyond this size call for methods without the need of (volume) meshing.
} 
However, outside of these above circumstances (in particular in the case of F.2 and F.3), a volume-discretization method inside $\Omega_{\text {hetero }}$ generates large linear systems due to its need for meshing ${ }^{6} 7$. To overcome these difficulties, we follow a method in the family BIE which has been used by 6 for Dirichlet condition and extended to other boundary conditions in [7], for configurations of up to 2500 obstacles of size $0.02 \lambda$. In this method, the scattered response is written as a superposition of waves scattered by each obstacle and expressed in the form of acoustic Single-Layer Potentials (SLPs), the Helmholtz equation and the radiation condition are thus satisfied automatically. When there are $N$ non-overlapping obstacles $\Omega_{I}$ with boundary denoted by $\Gamma_{I}, 1 \leq I \leq N$, we write

$$
u(x)=\sum_{I=1}^{N}\left(\mathcal{S}_{I} \tilde{v}_{I}\right)(x):=\sum_{I=1}^{N} \int_{\Gamma_{I}} G_{\kappa}(x, y) \tilde{v}_{I}(y) d y \quad, \quad \tilde{v}_{I} \in \mathcal{C}\left(\Gamma_{I}\right) .
$$

Here $G_{\kappa}$ is the outgoing fundamental solution at wavenumber $\kappa$, and is given in terms of the Hankel function of the first kind $\mathrm{H}_{0}^{(1)}$,

$$
G_{\kappa}(x, y):=\frac{\mathrm{i}}{4} \mathrm{H}_{0}^{(1)}(\kappa|x-y|) \quad, \quad x \neq y .
$$

The unknowns of the multi-scattering problem are now the single-layer densities $\tilde{v}_{J}$. The discretized scattered waves are obtained by a truncation of the Fourier

25 Series of the single-layer densities. An approximation order $\mathbf{m}$ of the method uses $2 \mathbf{m}+1$ Fourier nodes between $\mathbf{- m}$ and $\mathbf{m}$. For these reasons and convenience, we call the method (by a non standard name) Fourier Series single-layer (FSSL).

In a related approach, [8] uses a combination of double and single-layer 30 potentials for configurations of up to 400 obstacles of size $\lambda$. Using T-matrix, 9 studies scattering for up to 2000 obstacles of radius $\leq 1.6 \lambda$. More sophisticated methods (coupling BIE with FEM etc.) are considered for the scattering by a low number $(\sim 2,3)$ of medium-sized obstacles, c.f. [10, 11, 5]. For very small obstacles, there is the possibility of using asymptotic methods, c.f. [12, 13] and 35 the references therein. For extensive discussion on the use of integral equations and other methods in multiple-scattering, we refer to the book 14 with update [15]. For applications of multiple-scattering problem, see also [6, 7] and the references therein.

We choose to work with the single-layer operator $\mathcal{S}$, since it gives rise to simple integral equations among other choices of layer operators and modified ones, c.f. [16, 8. The intrinsic problem of $\mathcal{S}$ regarding invertibility (and hence instability) does not arise with small obstacles, e.g. when the exterior wavenumber

\footnotetext{
${ }^{6}$ In the case of small obstacles, the mesh needs to be refined around the obstacles in order to describe accurately their interaction with the incident wave. When there is a large number of them, the meshing of the obstacles may not be straightforward and requires some effort.

${ }^{7} \Omega_{\text {hetero }}$ can be quite large if there are lot of obstacles (even very small) that are spaced far apart, e.g. Experiment 5. When the obstacles are close to one another $(\operatorname{Exp} 4 \mathrm{c}), \Omega_{\text {hetero }}$ is around $40 \lambda$, however when they are remote from one another, $\Omega_{\text {ext }}$ extends to $146 \lambda$.
} 
$\kappa$ satisfies

$$
\kappa \times \underset{\text { radius }}{\text { Obstacle }}<2 \quad\left(\Leftrightarrow \underset{\text { radius }}{\text { Obstacle }}<\lambda \pi^{-1} \sim 0.3 \lambda\right),
$$

c.f. Remark 2. In general, apart from the set of Dirichlet eigenvalues, which 40 is a discrete and infinite subset of the positive real line, the linear problem obtained from using single-layer Ansatz is invertible, c.f. Prop2 2 For a theoretical study of the spectral and condition number of the single-layer operator, we refer to [17, 18. With the choice of Fourier Series basis, acceptable precision $\left(\sim 10^{-7}\right)$ can be obtained with low approximation order $(<4)$. As a result, the

45 linear systems generated by FSSL, whose unknowns are the generalized Fourier Series coefficients of the single-layer densities, however dens $8^{8}$ are generally small (compared to that produced by FEM at the same precision). For a selfcontained discussion, we compute the linear systems for all boundary conditions (Dirichlet, Neumann, and Impedance), with a study of their well-posedness and Fredholmness. These formulations are used for $C^{2}$ and convex obstacles.

For numerical experiments, we limit ourselves to the scattering of discshaped obstacles. In this geometry, a single-layer potential with Fourier Series basis as densities can be written as a superposition of multipoles; as a result, the linear systems have explicit expressions, c.f. Section 3 Despite the existence 55 of pieces of software for this type of simulation, e.g. the Matlab toolbox $\mu$-diff [19, see also [9] for a review of available packages, we would like to develop a high-performance computing software that can work on cluster:9? Our codes offer choices of the direct solvers (Mumps, Lapack and Scalapack), and GMRES (with restart) solvers [20] with various preconditioners. While they can be

${ }_{60}$ applied to general configurations of circular obstacles, we restrict our numerical tests to periodic ones of equal size $€^{10}$ inspired by I2M physical experiments, e.g. Numerical Result 3.

In literature, iterative solvers seem to be a more popular choice for resolving the multiple-scattering linear systems, e.g. 8, 7, 6, 21, 9. Most relevant to our work is the study done for random configurations in [7, 21, 6, which suggests that the GMRES solvers are much more effective than direct solver (Gauss elimination), with considerable lead in the case of more than 1000 obstacles, c.f. [21, Figure 4]. They also suggest that iterative solvers will maintain this robustness as the media become denser, i.e. with more obstacles and with the 70 obstacles close to one another. While Gauss elimination is not a very competitive representative of the robust direct family, this raises the question whether, for very large configurations, other direct solvers also perform as poorly compared to iterative ones, and whether parallelization and shared-memory architectures can narrow the performance gap. This question is posed, with the application of inverse problem ${ }^{11}$ in mind. For this reason, we carry out a detailed numerical

\footnotetext{
${ }^{8} \mathrm{~A}$ property shared in general by methods in the boundary integral equation family.

${ }^{9}$ This also adds flexibility to our work, which is part of a research program in collaboration with the Institut de Mécanique et d'Ingénierie at Bordeaux university (I2M).

${ }^{10}$ With the exception of Num. Exp.1. See Footnote 18 and 27

${ }^{11} \mathrm{An}$ example of this is reconstruction of location of small defects. For this type of appli-
} 
comparison between direct solvers (Mumps, Lapack and Scalapack) and iterative solvers, in Section 5, for closely-spaced and far-apart obstacles. The random configuration in [21, 6] corresponds more to our 'closely-spaced' experiments. The seven preconditioners in our study include two that are mentioned in [21, 6], the block Jacobi and one which is comparable to our 2nd-order Jacobi.

The paper is organized in two parts. The theoretical part studies the linear systems given by FSSL and their well-posedness in Section 2 3. The numerical comparisons are separated in two groups: those with Montjoie are in Section 4 and the solver comparison in Section 5 .

\section{Single-Layer Potential formulation of the multi scattering problem}

The multiple obstacle scattering problem of a time-harmonic incident wave is formulated as an exterior boundary-value problem (BVP) for impenetrable obstacles, i.e. where the wave is fully reflected. To write down the mathematical problems, we first fix the sign convention for the trace operators. The normal vector $n(x)$ along a smooth interface $\Gamma$ is chosen to point outward. The zero-th, first order traces for $f \in \mathcal{C}^{2}$ at $\Gamma$ is defined as: for $x \in \Gamma$,

$$
\begin{gathered}
\gamma_{0}^{+} f(x):=\lim _{h \rightarrow 0^{+}} f(x+h n(x)) \quad ; \quad \gamma_{0}^{-} f(x):=\lim _{h \rightarrow 0^{-}} f(x-h n(x)) ; \\
\gamma_{1}^{-} f(x):=\lim _{h \rightarrow 0^{+}} n(x) \cdot \nabla f(x-h n(x)) \\
\frac{\partial}{\partial n} f(x)=\gamma_{1}^{+} f(x):=\lim _{h \rightarrow 0^{+}} n(x) \cdot \nabla f(x+h n(x)) .
\end{gathered}
$$

We denote by $\Omega_{\text {ext }}$ the region outside of the obstacles, by $\Omega_{I}$ the region occupied by the $I$-th obstacle, and by $\Gamma_{I}$ its boundary, i.e. $\Gamma_{I}=\partial \overline{\Omega_{I}}$. The Boundary value problems we consider are the following.

$$
\left\{\begin{array}{lll}
\left(-\Delta-\kappa^{2}\right) u=0 & , \text { in } \Omega_{\text {ext }}, & \\
\gamma_{0, I}^{+}\left(u+u^{\mathbf{I}}\right)=0 & , 1 \leq I \leq N, & \text { Exterior Dirichlet } \\
\lim _{r \rightarrow \infty} \sqrt{r}\left(\partial_{r} u-\mathrm{i} \kappa u\right)=0 & , r=|x|, &
\end{array}\right.
$$

For the impedance problem, we denote by $\lambda$ the impedance parameter.

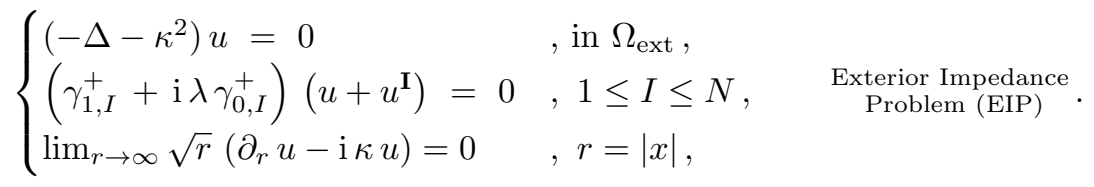

When $\lambda=0$, we have the Neumann problem (ENP). The Dirichlet and Neumann problems are also called soft and hard scattering problem, respectively.

The BVPs can be written as equivalent problems on a bounded domain, by replacing the outgoing radiation condition with an exact boundary condition

cation, it is preferable to use highly optimized direct solvers (Mumps, Lapack and Scalapack). See also Footnote 20 in Section 4 and Footnote 36 and 37 in Conclusion. 
using the Dirichlet-to-Neumann operator, c.f. [22, Lemma 5.24]. To show the existence and uniqueness of these equivalent problems, for Dirichlet problem, see 22, p.105], for impedance problem [23, Lemma 2.1] under the same assumption listed in in Theorem 4 in Appendix A. We note that the well-posedness results in 24] allow for multi-component interior domains, as in our setting, c.f. [24,

95 Section 2.1].

\subsection{Potential theory notations}

We list some facts of potential theory needed in our paper. We consider interfaces (along which the layer potentials are defined) that are closed, simple and $C^{2}$ curves. The acoustic single-layer potential along $\Gamma_{I}$ at wavenumber $\kappa$ and with density $\phi \in \mathcal{C}\left(\Gamma_{I}\right)$ is defined as,

$$
\left(\mathcal{S}_{I, \kappa} \phi\right)(x):=\int_{\Gamma_{I}} \phi(y) G_{\kappa}(x, y) d \sigma(y) \quad, \quad x \in \mathbb{R}^{2} \backslash \Gamma_{I} .
$$

Here $G_{\kappa}$ is the fundamental solution in (2). The single-layer potential defines outgoing solutions on $\mathbb{R}^{2} \backslash \Gamma_{I}$. It extends to the following map: for $-1 \leq s \leq 1$,

$$
\mathcal{S}_{I, \kappa}: H^{s-1 / 2}\left(\Gamma_{I}\right) \longrightarrow H_{\mathrm{loc}}^{s+1}\left(\mathbb{R}^{2}\right) \text { is bounded. }
$$

Remark 1. Since each obstacle is assumed to be simply-connected, we will follow [25, Section 8.2] to define the Sobolev spaces on its boundary. They are defined so that their pull-back to $[0,2 \pi)$ via a $2 \pi$-periodic parametrization of the boundary gives the usual Sobolev spaces on $[0,2 \pi)$.

To describe the traces of the layer potentials (along the interface where it is defined), we will need the following surface operators: for $x \in \Gamma_{I}, \phi \in \mathcal{C}\left(\Gamma_{I}\right)$,

$$
\begin{gathered}
\left(\mathrm{S}_{I, \kappa} \phi\right)(x):=\int_{\Gamma_{I}} \phi(y) G_{\kappa}(x, y) d s(y) ; \\
\left(\mathrm{D}_{I, \kappa}^{\prime} \phi\right)(x):=\int_{\Gamma_{I}} \phi(y) \frac{\partial}{\partial n(x)} G_{\kappa}(x-y) d s(y) .
\end{gathered}
$$

They extend to the following maps, c.f. [22, Theorem 7.3]: for $-1 \leq s \leq 1$

$$
\begin{gathered}
\mathrm{S}_{\kappa, I}: H^{s-1 / 2}\left(\Gamma_{I}\right) \longrightarrow H^{s+1 / 2}\left(\Gamma_{I}\right) \quad \text { is bounded and } \\
\mathrm{D}_{I, \kappa}^{\prime}: H^{s-1 / 2}\left(\Gamma_{I}\right) \longrightarrow H^{s-1 / 2}\left(\Gamma_{I}\right) \text { is compact and bounded. }
\end{gathered}
$$

We denote by $\left\{\gamma_{0, I}^{ \pm}, \gamma_{1, I}^{ \pm}\right\}$the traces (4) along the boundary $\Gamma_{I}$ of obstacle $I$. The traces of the single-layer potentials satisfy: for $-1 \leq s \leq 1$,

$$
\begin{gathered}
\gamma_{0, I}^{+} \mathcal{S}_{I, \kappa} \phi=\gamma_{0, I}^{-} \mathcal{S}_{I, \kappa} \phi=\mathrm{S}_{I, \kappa} \phi \quad, \quad \phi \in H^{-1 / 2+s}\left(\Gamma_{I}\right) ; \\
\gamma_{1, I}^{-} \mathcal{S}_{I, \kappa}=\mathrm{D}_{I, \kappa}^{\prime}+\frac{1}{2} \mathrm{Id} \quad, \quad \gamma_{1, I}^{+} \mathcal{S}_{I, \kappa}=\mathrm{D}_{I, \kappa}^{\prime}-\frac{1}{2} \mathrm{Id} \quad, \quad \phi \in H^{-1 / 2+s}\left(\Gamma_{I}\right) .
\end{gathered}
$$

To describe the multi-scattering problem, we will also need these following surface operators. Since the kernel of $\mathcal{S}_{I, \kappa}$ is smooth on $\mathbb{R}^{2} \backslash \Gamma_{I}$, for 
$\phi \in H^{-1 / 2}\left(\Gamma_{I}\right)$, we have $\mathcal{S}_{I, \kappa} \phi \in \mathcal{C}^{\infty}\left(\mathbb{R}^{2} \backslash \Gamma_{I}\right)$. As a result, the function and its normal derivative are continuous across $\Gamma_{J}$ for $J \neq I$. Thus, without having to distinguish between interior or exterior traces, we define

$$
\begin{aligned}
\mathrm{S}_{I J, \kappa} \phi:= & \gamma_{0, I} \mathcal{S}_{\kappa, J} \phi \quad, \quad \mathrm{D}_{I J}^{\prime} \phi:=\gamma_{1, I} \mathcal{S}_{\kappa, J} \phi, \\
& \text { for } \phi \in H^{-1 / 2}\left(\Gamma_{J}\right), I \neq J .
\end{aligned}
$$

\subsection{Linear systems}

As introduced, in the FSSL method, the scattered response is written as a superposition of waves scattered by each obstacle and expressed in the form of acoustic single-layer potentials,

$$
u=\sum_{J=1}^{N} \mathcal{S}_{J, \kappa} \tilde{v}_{J} \quad, \quad \tilde{v}_{J} \in H^{-1 / 2}\left(\Gamma_{J}\right) .
$$

This has the advantage that, as each single-layer potential satisfies the Helmholtz equation and the radiation condition, so does $u$, it thus remains to choose the densities $\tilde{v}_{J}$ so that the boundary conditions are satisfied. This is how the exterior problems (EDP, EIP) are reduced to equivalent problems the obstacle boundaries, and how the multiple-scattering linear systems are obtained. To label the BVPs, we write $\alpha=\mathrm{D}$ (Dirichlet), N (Neumann), and Im (Impedance).

We have denoted by $\left\{\gamma_{0, I}^{ \pm}, \gamma_{1, I}^{ \pm}\right\}$the family of traces along the boundary $\Gamma_{I}$ of obstacle $I$. Using the identities (9), we write the traces of the scattered wave $u$ along $\Gamma_{I}$ as

$$
\begin{gathered}
\gamma_{0, I}^{+} u=\sum_{J=1}^{N} \gamma_{0, I}^{+} \mathcal{S}_{J, \kappa} \tilde{v}_{J}=\sum_{J=1}^{N} \mathrm{~S}_{I J, \kappa} \tilde{v}_{J} \\
\gamma_{1, I}^{+} u=\sum_{J=1}^{N} \gamma_{1, I}^{+} \mathcal{S}_{J, \kappa} \tilde{v}_{J}=\left(\mathrm{D}_{I, \kappa}^{\prime}-\frac{1}{2} \mathrm{Id}\right) \tilde{v}_{I}+\sum_{J=1 ; J \neq I}^{N} \mathrm{D}_{I J, \kappa}^{\prime} \tilde{v}_{J} .
\end{gathered}
$$

For the following discussion, we assume that the incident wave $u^{\mathbf{I}}$ is smooth, and drop the distinction ' + ' and ' - ' from the notation of its traces, since they agree from both sides of a $\mathcal{C}^{2}$ interface.

Proposition 1. The BVPs (EDP, EIP, and ENP) are equivalent correspondingly to the following linear problems

$$
\mathbf{A}_{\alpha} \mathbf{V}=\mathbf{F}_{\alpha} \quad, \quad \mathbf{V}=\left(\tilde{v}_{J}\right)_{1 \leq J \leq N} \quad, \quad \mathbf{F}=\left(\mathbf{F}_{\alpha ; J}\right)_{1 \leq J \leq N},
$$

having as unknowns the single-layer densities $\mathbf{V}$. The coefficient operator is called the multi-scattering operator $\mathbf{A}_{\alpha}$ with

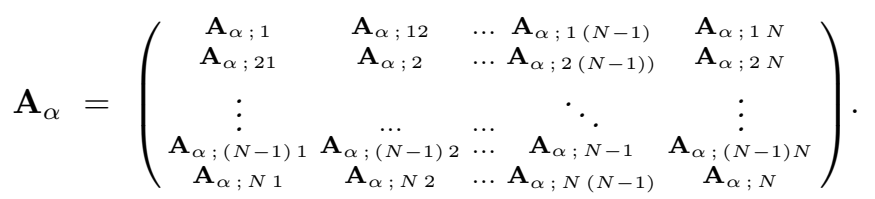


The diagonal operator $\mathbf{A}_{\alpha ; I}$, describing self-reflection by obstacle I, is given by

$$
\mathbf{A}_{\alpha ; I}= \begin{cases}\mathrm{S}_{I, \kappa} & , \alpha=D, \\ \mathrm{D}_{I, \kappa}^{\prime}-\frac{1}{2} \mathrm{Id}+\mathrm{i} \lambda \mathrm{S}_{I, \kappa} & , \alpha=I m, \\ \mathrm{D}_{I, \kappa}^{\prime}-\frac{1}{2} \mathrm{Id} & , \alpha=N .\end{cases}
$$

The off-diagonal $\mathbf{A}_{\alpha ; I J}$ with $I \neq J$ describes the diffraction by obstacle I wave emitted by $J$,

$$
\mathbf{A}_{\alpha ; I J}= \begin{cases}\mathrm{S}_{I J, \kappa} & , \alpha=D, \\ \mathrm{D}_{I J, \kappa}^{\prime}+\mathrm{i} \lambda \mathrm{S}_{I J, \kappa} & , \alpha=I m, \\ \mathrm{D}_{I J, \kappa}^{\prime} & , \alpha=N .\end{cases}
$$

The right hand side of (14) is defined in terms of the incident wave $u^{\mathbf{I}}$,

$$
\mathbf{F}_{\alpha ; I}=- \begin{cases}\gamma_{0, I} u^{\mathbf{I}} & , \alpha=D, \\ \left(\gamma_{1, I}+\mathrm{i} \lambda \gamma_{0, I}\right) u^{\mathbf{I}} & , \alpha=I m, \\ \gamma_{1, I} u^{\mathbf{I}} & , \alpha=N .\end{cases}
$$

Proof. Dirichlet problem : The scattered wave $u$ of the form (11) solves the EDP (5), if and only if $\left\{\tilde{v}_{I}\right\}$ satisfies $\gamma_{0, I}^{+} u=-\gamma_{0, I}^{+} u^{\mathbf{I}}$, for $1 \leq I \leq N$. Using $\sqrt{12}$, this equation is equivalent to

$$
\sum_{J=1}^{N} \mathrm{~S}_{I J, \kappa} \tilde{v}_{J}=-\gamma_{0, I} u^{\mathbf{I}} .
$$

Impedance problem : The scattered wave $u$ of the form 111 solves the EIP (6), if and only if $\left\{\tilde{v}_{I}\right\}$ satisfies correspondingly the following equations,

$$
\left(\gamma_{1, I}^{+}+\mathrm{i} \lambda \gamma_{0, I}^{+}\right) u=-\left(\gamma_{1, I}^{+}+\mathrm{i} \lambda \gamma_{0, I}^{+}\right) u^{\mathbf{I}}, 1 \leq I \leq N .
$$

Using $(12)$ and $(13)$, these above equations are equivalent to

$$
\left(\mathrm{D}_{I, \kappa}^{\prime}-\frac{1}{2} \mathrm{Id}+\mathrm{i} \lambda \mathrm{S}_{I, \kappa}\right) \tilde{v}_{I}+\sum_{J=1, J \neq I}\left(\mathrm{D}_{I J, \kappa}^{\prime}+\mathrm{i} \lambda \mathrm{S}_{I J, \kappa}\right) \tilde{v}_{J}=-\left(\gamma_{1, I}+\mathrm{i} \lambda \gamma_{0, I}\right) u^{\mathbf{I}} .
$$

When $\lambda=0$ in 190 , we obtain the Neumann problem.

\subsection{Mapping properties, Fredholmness and Invertibility}

In this section, we study the mapping properties of the multi-scattering operator $\mathbf{A}_{\alpha}$. Applying those of layer potentials (7) to the components of $\mathbf{A}_{\alpha}$, we obtain readily that

$$
\begin{aligned}
& \mathbf{A}_{\alpha}: \mathbb{H}_{1 / 2}\left(\boldsymbol{\Gamma}_{\text {Obs }}\right) \quad \longrightarrow \quad \mathbb{H}_{1 / 2}\left(\boldsymbol{\Gamma}_{\text {Obs }}\right), \quad \alpha=\operatorname{Im}, N \text {, } \\
& \mathbf{A}_{\alpha}: \mathbb{H}_{-1 / 2}\left(\boldsymbol{\Gamma}_{\text {Obs }}\right) \longrightarrow \mathbb{H}_{1 / 2}\left(\boldsymbol{\Gamma}_{\text {Obs }}\right), \quad \alpha=D,
\end{aligned}
$$


where

$$
\mathbb{H}_{s}\left(\boldsymbol{\Gamma}_{\text {Obs }}\right):=H^{s}\left(\Gamma_{1}\right) \times \ldots \times H^{s}\left(\Gamma_{N}\right) .
$$

Here $H^{s}\left(\Gamma_{I}\right)$ is the Sobolev space of order $s$ on the boundary of an obstacle $I$, see also Remark 1 . When $s=0$, we write $\mathbb{L}^{2}\left(\boldsymbol{\Gamma}_{\text {Obs }}\right)$. While the single-layer surface operator $\mathrm{S}$ is compact and is thus not invertible, we will show that apart from the set of Dirichlet eigenvalues, the linear problems (14) obtained from using single-layer Ansatz are well-posed. This is obtained by first showing that $\mathbf{A}_{\alpha}$ ${ }_{120}$ is Fredholm, see Lemma 5 in Appendix A. Secondly, we show $\mathbf{A}_{\alpha}$ is injective, if the exterior wavenumber $\kappa$ is not the square root of an interior Dirichlet eigenvalue, see Lemma 6 in Appendix A. By Fredholmness, invertibility follows from injectivity, and we obtain

Proposition 2. If $\kappa^{2}$ is not a Dirichlet eigenvalue for $-\Delta$ inside domain $\Omega_{I}$ with $1 \leq 1 \leq N$, then $\mathbf{A}_{\alpha}$ is invertible, with $\alpha=D, N$ and Im.

Remark 2 (Small obstacles). Since our applications focus on very small obstacles, it would be useful to know numerically how small the obstacles should be, in order to be in the region of invertibility.

1. For a disc domain of radius $R$, the Dirichlet eigenvalues (DEV) are

$$
\lambda_{n, m}=\left(\frac{j_{n, m}}{R}\right)^{2} \quad, \quad n \geq 0 \quad, \quad m \geq 1 .
$$

Here, $j_{n, m}$ the $m$-th positive root of $\mathrm{J}_{n}(\cdot)=0$, and $\mathrm{J}_{n}$ is the Bessel function of the first kind at integer order $n$, for definition c.f. [26, $p$. 355]. The first 4 roots are

$$
j_{0,1} \sim 2.40 \quad, \quad j_{1,1} \sim 3.83 \quad, \quad j_{2,1} \sim 5.13 \quad, \quad j_{1,2} \sim 5.52 .
$$

For invertibility in the case of circular obstacles, we require

$$
\kappa \times(\text { Obs radius }) \neq j_{n, m} .
$$

2. For general domains, the (Rayleigh-Faber-Krahn) Isoperimetric inequality gives the lower bound of the first interior DEV,

$$
\lambda_{1}(\Omega)=\inf _{u \in H_{0}^{1}(\Omega) \backslash\{0\}} \frac{\int_{\Omega}|\nabla u|^{2} d x}{\int_{\Omega} u^{2} d x} \geq \frac{\pi}{|\Omega|} j_{0,1}^{2},|\Omega|=\text { area of } \Omega .
$$

Equality is attained if and only if the membrane is circular. As a result, if $\kappa$ satisfies

$$
\kappa \times R(\Omega)<2, R(\Omega)=\text { the radius of } \Omega \text {, }
$$

then $\kappa^{2}$ is not a DEV on $\Omega$. This is due to

$$
j_{0,1} \sim 2.40>2 \quad \text { and } \quad \kappa^{2}<\frac{\pi \times 2^{2}}{\pi R(\Omega)}<\frac{\pi \times 2^{2}}{|\Omega|}<\frac{\pi}{|\Omega|} j_{0,1}^{2}<\lambda_{1}(\Omega) .
$$




\subsection{Variational forms and general discrete problems}

For $\alpha=\mathrm{D}$, from the proof of Lemma 5 in Appendix A, we obtain the decomposition $\mathbf{A}_{\alpha}=\tilde{\mathbf{A}}_{\alpha}+\mathbf{K}_{\alpha}$, where $\tilde{\mathbf{A}}_{\alpha}$ is coercive with respect to $\mathbb{H}_{-1 / 2}\left(\boldsymbol{\Gamma}_{\text {Obs }}\right)$, and $\tilde{\mathbf{A}}_{\alpha}: \mathbb{H}_{-1 / 2}\left(\boldsymbol{\Gamma}_{\text {Obs }}\right) \rightarrow \mathbb{H}_{1 / 2}\left(\boldsymbol{\Gamma}_{\text {Obs }}\right)$ is bounded and invertible, while $\mathbf{K}_{\alpha}$ : $\mathbb{H}_{-1 / 2}\left(\boldsymbol{\Gamma}_{\text {Obs }}\right) \rightarrow \mathbb{H}_{-1 / 2}\left(\boldsymbol{\Gamma}_{\text {Obs }}\right)$ is bounded and compact. Each of these operators corresponds to a sesquilinear form as follows,

$$
\begin{array}{ll}
\mathbf{a}_{\alpha}: \mathbb{H}_{-1 / 2}\left(\boldsymbol{\Gamma}_{\text {Obs }}\right) \times \mathbb{H}_{-1 / 2}\left(\boldsymbol{\Gamma}_{\text {Obs }}\right) \rightarrow \mathbb{C} & ; \mathbf{a}_{\alpha}(\boldsymbol{\psi}, \boldsymbol{\phi}):=\left\langle\mathbf{A}_{\alpha} \boldsymbol{\psi}, \boldsymbol{\phi}\right\rangle_{\mathbb{H}_{1 / 2}, \mathbb{H}_{-1 / 2}} ; \\
\tilde{\mathbf{a}}_{\alpha}: \mathbb{H}_{-1 / 2}\left(\boldsymbol{\Gamma}_{\text {Obs }}\right) \times \mathbb{H}_{-1 / 2}\left(\boldsymbol{\Gamma}_{\text {Obs }}\right) \rightarrow \mathbb{C} & ; \tilde{\mathbf{a}}_{\alpha}(\boldsymbol{\psi}, \boldsymbol{\phi}):=\left\langle\tilde{\mathbf{A}}_{\alpha} \boldsymbol{\psi}, \boldsymbol{\phi}\right\rangle_{\mathbb{H}_{1 / 2}, \mathbb{H}_{-1 / 2}} ; \\
\mathbf{k}_{\alpha}: \mathbb{H}_{-1 / 2}\left(\boldsymbol{\Gamma}_{\text {Obs }}\right) \times \mathbb{H}_{-1 / 2}\left(\boldsymbol{\Gamma}_{\text {Obs }}\right) \rightarrow \mathbb{C} & ; \mathbf{k}_{\alpha}(\boldsymbol{\psi}, \boldsymbol{\phi}):=\left\langle\mathbf{K}_{\alpha} \boldsymbol{\psi}, \boldsymbol{\phi}\right\rangle_{\mathbb{H}_{1 / 2}, \mathbb{H}_{-1 / 2}} .
\end{array}
$$

We have $\mathbf{a}_{\alpha}=\tilde{\mathbf{a}}_{\alpha}+\mathbf{k}_{\alpha}$.

Similarly, for $\alpha=\operatorname{Im}$ and $\mathrm{N}$, we obtain the decomposition $\mathbf{A}_{\alpha}=\frac{1}{2} \mathbf{I d}+\mathbf{K}_{\alpha}$, with corresponding forms $\mathbf{a}_{\alpha}$ and $\mathbf{k}_{\alpha}$,

$$
\begin{aligned}
& \mathbf{a}_{\alpha}, \mathbf{k}_{\alpha}: \mathbb{H}_{1 / 2}\left(\boldsymbol{\Gamma}_{\text {Obs }}\right) \times \mathbb{H}_{-1 / 2}\left(\boldsymbol{\Gamma}_{\text {Obs }}\right) \longrightarrow \mathbb{C} \\
& \mathbf{a}_{\alpha}(\boldsymbol{\psi}, \boldsymbol{\phi}):=\frac{1}{2}\langle\boldsymbol{\psi}, \boldsymbol{\phi}\rangle_{\mathbb{H}_{1 / 2}, \mathbb{H}_{-1 / 2}}+\mathbf{k}_{\alpha}(\boldsymbol{\psi}, \boldsymbol{\phi}) .
\end{aligned}
$$

We define the linear function corresponding to the RHS $\mathbf{F}_{\alpha}$,

$$
\begin{gathered}
\boldsymbol{\ell}_{\alpha}: \mathbb{H}_{-1 / 2}\left(\boldsymbol{\Gamma}_{\text {Obs }}\right) \longrightarrow \mathbb{C} \\
\boldsymbol{\ell}_{\alpha}(\boldsymbol{\varphi})=\left\langle\mathbf{F}_{\alpha}, \boldsymbol{\varphi}\right\rangle_{\mathbb{H}_{1 / 2}, \mathbb{H}_{-1 / 2}}=\sum_{I=1}^{N}\left\langle\mathbf{F}_{\alpha, I}, \varphi_{I}\right\rangle_{H^{1 / 2}\left(\Gamma_{I}\right), H^{-1 / 2}\left(\Gamma_{I}\right)}
\end{gathered}
$$

As a result, the problems 14 can be put in the following variational form,

$$
\begin{gathered}
\text { For } \mathbf{F}_{\alpha} \in \mathbb{H}_{1 / 2}\left(\boldsymbol{\Gamma}_{\text {Obs }}\right), \text { find } \boldsymbol{\psi}_{\alpha} \in \begin{cases}\mathbb{H}_{-1 / 2}\left(\boldsymbol{\Gamma}_{\text {Obs }}\right) & \text { for } \alpha=D, \\
\mathbb{H}_{1 / 2}\left(\boldsymbol{\Gamma}_{\text {Obs }}\right) & \text { for } \alpha=\operatorname{Im}, N\end{cases} \\
\text { such that } \mathbf{a}_{\alpha}(\boldsymbol{\psi}, \boldsymbol{\varphi})=\boldsymbol{\ell}_{\alpha}(\boldsymbol{\varphi}) \quad, \quad \forall \boldsymbol{\varphi} \in \mathbb{H}_{-1 / 2}\left(\boldsymbol{\Gamma}_{\text {Obs }}\right) .
\end{gathered}
$$

The discretization of the variational problem $(23)$ is via a dense sequence of finite-dimensional spaces in $\mathbb{H}_{-1 / 2}\left(\boldsymbol{\Gamma}_{\text {Obs }}\right)$ (for $\alpha=D$ ) or $\mathbb{H}_{1 / 2}\left(\boldsymbol{\Gamma}_{\text {Obs }}\right)$ (for $\alpha=\operatorname{Im}, N)$. Let $\left\{V_{l}\right\}_{l \in \mathbb{N}}$ be a dense sequence of finite-dimensional subspaces in $H^{-1 / 2}(\Gamma)$ for $\alpha=D$, and $H^{1 / 2}(\Gamma)$ for $\alpha=\operatorname{Im}, N$. Write

$$
\mathbb{V}_{\mathbf{m}}:=V_{\mathbf{m}}\left(\Gamma_{1}\right) \times \ldots \times V_{\mathbf{m}}\left(\Gamma_{N}\right) .
$$

With $\boldsymbol{\ell}_{\alpha}$ defined in $(22)$, the discretized version of the problem (23) for approximation of order $\mathbf{m}$ is written as :

For $\mathbf{F}_{\alpha} \in \mathbb{H}_{1 / 2}\left(\boldsymbol{\Gamma}_{\text {Obs }}\right)$, find $\boldsymbol{\psi}_{h} \in \mathbb{V}_{\mathbf{m}}$

$$
\text { such that } \mathbf{a}_{\alpha}\left(\psi_{h}, \varphi\right)=\ell_{\alpha}(\varphi) \quad, \quad \forall \varphi \in \mathbb{V}_{\mathbf{m}} .
$$

Remark 3 (General well-posedness of the discrete problem). The above decompositions of $\mathbf{a}_{\alpha}$ satisfy the hypothesis of [27, Thm 4.2.9,p.229]. As a result, the well-posedness of the approximate problems (24) are obtained, when injectivity is satisfied. The latter is guaranteed when $\kappa^{2}$ is not an interior DEV for $\Omega_{I}$, $1 \leq I \leq N$, c.f. Lemma 6. Results in [27, Thm 4.2.9,p.229] also give a Céa-type estimate for the approximation error. 


\subsection{Fourier Series Galerkin Basis}

We can write the single-layer densities in terms of the Fourier series on the boundary of the obstacles. Fix a $\mathcal{C}^{2} 2 \pi$-periodic parametrization $\phi_{I}$ of $\Gamma_{I}$ (boundary of obstacle I), the basis functions $\mathbf{w}_{I, k}$ on $\Gamma_{I}$ are defined $\sqrt{12}$ so that

$$
\phi_{I}:[0,2 \pi) \rightarrow \Gamma_{I} \subset \mathbb{R}^{2} \quad, \quad\left(\mathbf{w}_{I, k} \circ \phi_{I}\right)(\theta)=e^{\mathrm{i} k \theta} .
$$

As a result, their linear combinations with complex coefficients, when pulled back to $[0,2 \pi)$ via $\phi_{J}$, give the set of trigonometric polynomials on $[0,2 \pi)$,

$$
\bigcup_{\mathbf{m} \in \mathbb{N}}\left\{\sum_{k=-\mathbf{m}}^{\mathbf{m}} a_{k} e^{\mathrm{i} k \theta}, a_{k} \in \mathbb{C}\right\} .
$$

Note this set is dense in $H^{r}(0,2 \pi)$ for all $r \in \mathbb{R}$.

FSSL method. The exact diffracted wave $u$ and approximate $u_{h}$ are written in this basis with the Fourier coefficients $\mathbf{V}=\left(V_{J, k}\right)_{1 \leq J \leq N, k \in \mathbb{Z}}$ as

$$
u_{h}=\sum_{J=1}^{N} \mathcal{S}_{J} \sum_{k=-\mathbf{m}}^{\mathbf{m}} V_{J, k} \mathbf{w}_{J, k} \stackrel{\mathbb{L}^{2}\left(\boldsymbol{\Gamma}_{\text {Obs }}\right)}{\longrightarrow} u=\sum_{J=1}^{N} \mathcal{S}_{J} \sum_{k=-\infty}^{\infty} V_{J, k} \mathbf{w}_{J, k} .
$$

The convergence is in norm $\|\cdot\|_{\mathbb{L}^{2}\left(\boldsymbol{\Gamma}_{\mathrm{Obs}}\right)}$, defined in 21$)$. Substituting the above form of $u$ and $u_{h}$ in (23), we obtain the linear system for $\left\{V_{J, k}\right\}$,

$$
\sum_{J=1}^{N} \sum_{k=-\mathbf{m}}^{\mathbf{m}} V_{J, k} \mathbf{a}_{\alpha}\left(\mathcal{S}_{J} \mathbf{w}_{J, k}, \mathbf{w}_{I, l}\right)=\boldsymbol{\ell}_{\alpha}\left(\mathbf{w}_{I, l}\right), \forall 1 \leq I \leq N, l \in \mathbb{Z} .
$$

Recall that $\boldsymbol{\ell}_{\alpha}$ is defined in 22), corresponding to $\mathbf{F}_{\alpha} \in \mathbb{H}_{1 / 2}\left(\boldsymbol{\Gamma}_{\text {Obs }}\right)$ in (17). As a result, the multi-scattering problem (14) can be written as

$$
\mathbf{A}_{\alpha} \mathbf{V}=\mathbf{F}_{\alpha} \quad, \quad \mathbf{V}=\left(V_{J, k}\right)_{1 \leq J \leq N, k \in \mathbb{Z}} .
$$

Although we use the same notations as in 14 , the multi-scattering operator $\mathbf{A}_{\alpha}$ is now an infinite matrix, composed of $N \times N$ blocks, each being an infinite matrix. Similarly, the RHS is composed of $N$ blocks, with each being an infinite vector. Their components are given by

$$
\begin{gathered}
\left(\mathbf{A}_{\alpha, I J}\right)_{l k}:=\mathbf{a}_{\alpha}\left(\mathcal{S}_{J} \mathbf{w}_{J, k}, \mathbf{w}_{I, l}\right) \quad, \quad\left(\mathbf{F}_{\alpha}\right)_{I l}:=\ell_{\alpha}\left(\mathbf{w}_{I, l}\right) \\
1 \leq I, J \leq N, k, l \in \mathbb{Z}
\end{gathered}
$$

The discretized problem at order $\mathbf{m}$ is a linear system of size $2 \mathbf{m}+1$

$$
\mathbf{A}_{\alpha, h} \mathbf{V}_{h}=\mathbf{F}_{\alpha, h} \quad, \quad \mathbf{V}_{h}=\left(V_{J, k}\right)_{1 \leq J \leq N,-\mathbf{m} \leq k \leq \mathbf{m}},
$$

\footnotetext{
${ }^{12}$ This is related to the idea of using $2 \pi$-periodic parametrization to define Sobolev spaces on the boundary of the obstacles, see Remark 1
} 
where $\mathbf{A}_{\alpha, h}$ is a truncated version of $\mathbf{A}_{\alpha}$, composed of $N \times N$ blocks, each block being a matrix of size $(2 \mathbf{m}+1) \times(2 \mathbf{m}+1)$, and $\mathbf{F}_{\alpha, h}$ is a truncated version of $\mathbf{F}_{\alpha}$ composed of $N$ blocks, each being a vector of size $2 \mathbf{m}+1$,

$$
\begin{gathered}
\left(\mathbf{A}_{\alpha, h, I J}\right)_{l k}:=\mathbf{a}_{\alpha}\left(\mathcal{S}_{J} \mathbf{w}_{J, k}, \mathbf{w}_{I, l}\right) \quad, \quad-\mathbf{m} \leq k, l \leq \mathbf{m}, 1 \leq I, J \leq N ; \\
\left(\mathbf{F}_{\alpha, h}\right)_{I l}:=\ell_{\alpha}\left(\mathbf{w}_{I, l}\right) \quad, \quad-\mathbf{m} \leq l \leq \mathbf{m}, 1 \leq I \leq N .
\end{gathered}
$$

For convenience, we refer to this method as Fourier Series - Single Layer (FSSL).

${ }_{140}$ Remark 4 (Convergence). The above approximation is a case of a projection method. Hence, in addition to Remark 3, another general error analysis can be obtained from projection theory, c.f. [16, Section 5].

\section{Multiple scattering for the disc-shaped obstacles}

When the obstacles (denoted by $\Omega_{I}, 1 \leq I \leq N$ ) are circular, the singlelayer potential with the basis element $\mathbf{w}_{J, l}$ as density and its traces have explicit descriptions in terms of multipoles, c.f. 32 - (36). We will use these expansions to calculate explicitly the components of the linear systems (29). They use Bessel functions and Hankel functions of the first kind $\mathrm{J}_{l}$ and $\mathrm{H}_{l}^{(1)}$, see definition e.g. in [26, p. 355].

We assume the circular obstacle $I$ is of radius $\mathrm{r}_{I}$ and centered at $\mathbf{x}_{I} \in \mathbb{R}^{2}$. Denote the polar coordinates relative to $\mathbf{x}_{I}$ by $\left(r_{I}(\cdot), \theta_{I}(\cdot)\right)$,

$$
x=\mathbf{x}_{I}+r_{I}(x)\left(\cos \theta_{I}(x), \sin \theta_{I}(x)\right) .
$$

Denote by $\mathrm{d}_{I J}$ the distance between the centers of obstacle $I$ and $J$, and by $\theta_{I J}$ and $\theta_{J I}$ their relative polar coordinates,

$$
\mathbf{x}_{I}=\mathbf{x}_{J}+\mathrm{d}_{I J}\left(\cos \theta_{J I}, \sin \theta_{J I}\right) ; \quad \mathbf{x}_{J}=\mathbf{x}_{I}+\mathrm{d}_{I J}\left(\cos \theta_{I J}, \sin \theta_{I J}\right) .
$$

The non-overlapping assumption is given by $\mathrm{d}_{I J}>\mathrm{r}_{I}+\mathrm{r}_{J}$.

In this section, we remove $\kappa$ from the notation of the single-layer potential, and simply write $\mathcal{S}_{J}$. For $I \neq J$, at points $x$ such that $r_{I}(x)<\mathrm{d}_{I J}$, the singlelayer potential $\mathcal{S}_{J} \mathbf{w}_{J, l}$, in polar coordinates relative to $\mathbf{x}_{I}$, is given by

$$
\begin{gathered}
\left(\mathcal{S}_{J} \mathbf{w}_{J, l}\right)\left(r_{I}(x), \theta_{I}(x)\right)= \\
\frac{\mathrm{i} \pi \mathrm{r}_{J}}{2} \mathrm{~J}_{l}\left(\kappa \mathrm{r}_{J}\right) \sum_{m=-\infty}^{\infty} \mathrm{H}_{l-m}^{(1)}\left(\kappa \mathrm{d}_{I J}\right) e^{\mathrm{i}(l-m) \theta_{J I}} \mathrm{~J}_{m}\left(\kappa r_{I}(x)\right) e^{\mathrm{i} m \theta_{I}(x)} .
\end{gathered}
$$

Taking the exterior zero-th and first order traces along $\Gamma_{I}$,

$$
\begin{gathered}
\left(\gamma_{0, I}^{+} \mathcal{S}_{J} \mathbf{w}_{J, l}\right)\left(\theta_{I}(x)\right)= \\
\frac{\mathrm{i} \pi \mathrm{r}_{J}}{2} \mathrm{~J}_{l}\left(\kappa \mathrm{r}_{J}\right) \sum_{m=-\infty}^{\infty} \mathrm{H}_{l-m}^{(1)}\left(\kappa \mathrm{d}_{I J}\right) e^{\mathrm{i}(l-m) \theta_{J I}} \mathrm{~J}_{m}\left(\kappa \mathrm{r}_{I}\right) e^{\mathrm{i} m \theta_{I}(x)},
\end{gathered}
$$


and

$$
\begin{gathered}
\left(\gamma_{1, I}^{+} \mathcal{S}_{J} \mathbf{w}_{J, l}\right)\left(\theta_{I}(x)\right)= \\
\frac{\mathrm{i} \pi \mathrm{r}_{J} \kappa}{2} \mathrm{~J}_{l}\left(\kappa \mathrm{r}_{J}\right) \sum_{m=-\infty}^{\infty} \mathrm{H}_{l-m}^{(1)}\left(\kappa \mathrm{d}_{I J}\right) e^{\mathrm{i}(l-m) \theta_{J I}} \mathrm{~J}_{m}^{\prime}\left(\kappa \mathrm{r}_{I}\right) e^{\mathrm{i} m \theta_{I}(x)} .
\end{gathered}
$$

For $I=J$, for points $x \notin \Omega_{I}$, the single-layer potential $\mathcal{S}_{J} \mathbf{w}_{J, l}$ in polar coordinates relative to $\mathbf{x}_{I}$, is given by,

$$
\left(\mathcal{S}_{J} \mathbf{w}_{J, l}\right)\left(r_{I}(x), \theta_{I}(x)\right)=\frac{\mathrm{i} \pi \mathrm{r}_{J}}{2} e^{\mathrm{i} l \theta_{J}(x)} \mathrm{J}_{l}\left(\kappa \mathrm{r}_{J}\right) \mathrm{H}_{l}^{(1)}\left(\kappa r_{J}(x)\right) .
$$

Taking the exterior zero-th and first order traces along $\Gamma_{I}(I=J)$,

$$
\begin{gathered}
\left(\gamma_{0, I}^{+} \mathcal{S}_{J} \mathbf{w}_{J, l}\right)\left(\theta_{I}(x)\right)=\frac{i \pi \mathrm{r}_{J}}{2} e^{\mathrm{i} l \theta_{J}(x)} \mathrm{J}_{l}\left(\kappa \mathrm{r}_{J}\right) \mathrm{H}_{l}^{(1)}\left(\kappa \mathrm{r}_{J}\right) ; \\
\left(\gamma_{1, I}^{+} \mathcal{S}_{J} \mathbf{w}_{J, l}\right)\left(\theta_{I}(x)\right)=\frac{\mathrm{i} \pi \mathrm{r}_{J} \kappa}{2} e^{\mathrm{i} l \theta_{J}(x)} \mathrm{J}_{l}\left(\kappa \mathrm{r}_{J}\right) \mathrm{H}_{l}^{(1) \prime}\left(\kappa \mathrm{r}_{J}\right) .
\end{gathered}
$$

See [28, Appendix B3, B4] for their derivation.

As incident wave, we consider a plane wave with incident angle $\alpha_{\text {inc }}$,

$$
u_{\mathbf{p w}}(x)=e^{\mathrm{i} \kappa x \cdot\left(\cos \alpha_{\mathrm{inc}}, \sin \alpha_{\mathrm{inc}}\right)} .
$$

Using (31), we can write this in terms of the polar coordinates with respect to obstacle $\mathbf{x}_{I}$,

$$
u_{\mathbf{p w}}(x)=u_{\mathbf{p w}}\left(\mathbf{x}_{I}\right) e^{\mathrm{i} \kappa r_{I}(x) \cos \left(\theta_{I}(x)-\alpha_{\mathrm{inc}}\right)} .
$$

The exponential term is developed using the Jacobi expansion [14, Eq. 2.17]

$$
u_{\mathbf{p w}}(x)=u_{\mathbf{p w}}\left(\mathbf{x}_{I}\right) \sum_{l=-\infty}^{\infty} \mathrm{i}^{l} \mathrm{~J}_{l}\left(\kappa r_{I}(x)\right) e^{\mathrm{i} l\left(\theta_{I}(x)-\alpha_{\mathrm{inc}}\right)} .
$$

Taking the exterior zero-th and first order traces along $\Gamma_{I}$,

$$
\begin{aligned}
& \left(\gamma_{0, I}^{+} u_{\mathbf{p w}}\right)\left(\theta_{I}(x)\right)=u_{\mathbf{p w}}\left(\mathbf{x}_{I}\right) \sum_{l=-\infty}^{\infty} \mathrm{i}^{l} \mathrm{~J}_{l}\left(\kappa \mathrm{r}_{I}\right) e^{\mathrm{i} l\left(\theta_{I}(x)-\alpha_{\mathrm{inc}}\right)} \\
& \left(\gamma_{1, I}^{+} u_{\mathbf{p w}}\right)\left(\theta_{I}(x)\right)=\kappa u_{\mathbf{p w}}\left(\mathbf{x}_{I}\right) \sum_{l=-\infty}^{\infty} \mathrm{i}^{l} \mathrm{~J}_{l}^{\prime}\left(\kappa \mathrm{r}_{I}\right) e^{\mathrm{i} l\left(\theta_{I}(x)-\alpha_{\mathrm{inc}}\right)}
\end{aligned}
$$

These above expansions are the main ingredients to arrive at the linear system for the multiple-scattering of circular obstacles, for more details of the proof, see [28, Section 4.1].

Proposition 3 (Circular Obstacles). The exact scattered wave $u$ and the approximate one $u_{h}$ at order $\mathbf{m}$ given by FSSL method are

$$
u(x):=\sum_{I=1}^{\mathbf{N}} \sum_{k=-\infty}^{\infty} V_{I, k}\left(\mathcal{S}_{I} \mathbf{w}_{I, k}\right)(x) ; u_{h}(x):=\sum_{I=1}^{\mathbf{N}} \sum_{k=-\mathbf{m}}^{\mathbf{m}} V_{I, k}\left(\mathcal{S}_{I} \mathbf{w}_{I, k}\right)(x) ;
$$


with

$$
\left(\mathcal{S}_{I} \mathbf{w}_{I, k}\right)\left(r_{I}(x), \theta_{I}(x)\right)=\frac{\mathrm{i} \pi \mathrm{r}_{I}}{2} e^{\mathrm{i} k \theta_{I}(x)} \mathrm{J}_{k}\left(\kappa \mathrm{r}_{I}\right) \mathrm{H}_{k}^{(1)}\left(\kappa r_{I}(x)\right) .
$$

The unknowns for the exact solution are the Fourier coefficients of the single-

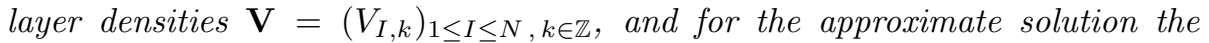

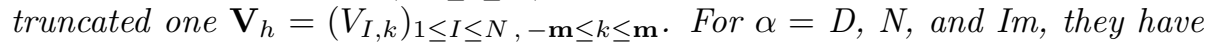
to satisfy the following linear system

$$
\mathbf{A}_{\alpha} \mathbf{V}=\mathbf{F}_{\alpha} \quad ; \quad \mathbf{A}_{\alpha, h} \mathbf{V}_{h}=\mathbf{F}_{\alpha, h} .
$$

The coefficient matrix $\mathbf{A}_{\alpha}$ is composed of $N \times N$ blocks. Its diagonal blocks $\mathbf{A}_{\alpha, I}$ are diagonal infinite matrices, with diagonal components given by

$$
\left(\mathbf{A}_{\alpha, I}\right)_{l l}=\frac{\mathrm{i} \pi \mathrm{r}_{I} \mathrm{~J}_{l}\left(\kappa \mathrm{r}_{I}\right)}{2} \times \begin{cases}\mathrm{H}_{l}^{(1)}\left(\kappa \mathrm{r}_{I}\right) & , \alpha=D \\ \kappa \mathrm{H}_{l}^{(1) \prime}\left(\kappa \mathrm{r}_{I}\right) & , \alpha=N \\ \mathrm{i} \lambda \mathrm{H}_{l}^{(1)}\left(\kappa \mathrm{r}_{I}\right)+\kappa \mathrm{H}_{l}^{(1) \prime}\left(\kappa \mathrm{r}_{I}\right) & , \alpha=I m\end{cases}
$$

For $I \neq J$, the components of the off-diagonal block $\mathbf{A}_{\alpha, I J}$ are given by,

$$
\begin{aligned}
\left(\mathbf{A}_{\alpha, I J}\right)_{l m}=\frac{\mathrm{i} \pi \mathrm{r}_{J}}{2} \mathrm{~J}_{m}\left(\kappa \mathrm{r}_{J}\right) \mathrm{H}_{m-l}^{(1)}\left(\kappa \mathrm{d}_{I J}\right) e^{\mathrm{i}(m-l) \theta_{J I}} & \\
& \times \begin{cases}\mathrm{J}_{l}\left(\kappa \mathrm{r}_{I}\right) & , \alpha=D \\
\kappa \mathrm{J}_{l}^{\prime}\left(\kappa \mathrm{r}_{I}\right) & , \alpha=N . \\
\mathrm{i} \lambda \mathrm{J}_{l}\left(\kappa \mathrm{r}_{I}\right)+\kappa \mathrm{J}_{l}^{\prime}\left(\kappa \mathrm{r}_{I}\right) & , \alpha=I m\end{cases}
\end{aligned}
$$

For the scattering of the plane wave (37), the components of the RHS of 42 are given by

$$
\left(F_{\alpha}\right)_{I, l}=-u_{p w}\left(\mathbf{x}_{I}\right) \mathrm{i}^{l} e^{-\mathrm{i} l \alpha_{i n c}} \times \begin{cases}\mathrm{J}_{l}\left(\kappa \mathrm{r}_{I}\right) & , \alpha=D, \\ \kappa \mathrm{J}_{l}^{\prime}\left(\kappa \mathrm{r}_{I}\right) & , \alpha=N, \\ \mathrm{i} \lambda \mathrm{J}_{l}\left(\kappa \mathrm{r}_{I}\right)+\kappa \mathrm{J}_{l}^{\prime}\left(\kappa \mathrm{r}_{I}\right) & , \alpha=I m .\end{cases}
$$

The coefficient matrix $\mathbf{A}_{\alpha, h}$ and the $R H S \mathbf{F}_{\alpha, h}$ of the approximate problem are truncated versions of the exact ones, as given in (30).

Remark 5. 1. The above results can be rewritten entirely using Multipole Theory without mentioning single-layer potentials, see e.g. [28, Remark $5]$.

2. For a fixed wavelength and small enough obstacles, it is expected that the rate of convergence increases as the radius of the obstacles and the distance between them reduce, and decreases with increasing number of the obstacles. For more details, see [28, Section 4.3]. 


\section{Validation of codes and comparison with Montjoie}

165 direct solver Mumps or Lapack) and the software Montjoie (MJ). These serve to validate the observed shortcomings of a volume FE-based method for configurations with one or a combination of the factors F.1-F.3 defined in the Introduction. The numerical results of FSSL were generated by codes written in Fortran90, and those by Montjoie codes in $\mathrm{C}++$, both with double precision and a parallel architecture ${ }^{13}$. Montjoie computes the solution of Helmholtz equation in a finite domain bounded with Perfectly Matched Layers, on a quadrangular mesh refined around the obstacles. Its bases are Lagrange spectral high order curved finite elements based on Gauss-Lobatto points. The inversion of the lin-

175 ear system uses Mumps. In the following discussion (concerning Montjoie), we write QN to denote the N-th order FE on quadrangular meshes.

To evaluate the time costs, we distinguish between pre-processing (used for construction and resolution of linear systems of unknowns), and post-processing time (to evaluate the diffracted wave on a visualization grid). For FSSL, preprocessing solves the linear systems (42) for unknowns $\left(V_{I, k}\right)$, which are the Fourier coefficients of the single-layer densities on the boundary of each obstacle, while post-processing evaluates the diffracted field of the form,

$$
\sum_{I=1}^{N_{\text {Obs }}} \frac{\mathrm{i} \pi \mathrm{r}_{I}}{2} \sum_{k=-\mathbf{m}}^{\mathbf{m}} V_{I k} e^{\mathrm{i} k \theta_{I}(x)} \mathrm{J}_{k}\left(\kappa \mathrm{r}_{I}\right) \mathrm{H}_{k}^{(1)}\left(\kappa\left\|x-\mathbf{x}_{I}\right\|\right),
$$

for each point in the visualization grid. To compare the post-processing results (the approximate total or diffracted wave on the domain of visualization or at receivers) between FSSL and Montjoie, we use (discrete) $L^{2}$ norms. All results obtained by Montjoie can only be compared via this norm. On the other hand, for results obtained by FSSL, and for comparisons within FSSL (e.g. among different orders of discretization to obtain convergence curves, see discussion below, or to compare among different solvers as done in Section 5), we only have to compare the pre-processing results (the single-layer densities) in the norm ${ }^{14} \mathbb{H}_{1 / 2}=\mathbb{H}_{1 / 2}\left(\boldsymbol{\Gamma}_{\text {Obs }}\right)$ defined in (21). For circular obstacles, this norm can be written as

$$
\left\|\mathbf{V}_{h}\right\|_{\mathbb{H}_{s}}=\sum_{I=1}^{N} \sum_{k=-\mathbf{m}}^{\mathbf{m}}\left|V_{I, k}\right|^{2}\left(1+k^{2}\right)^{s}, \mathbf{V}_{h}=\left(V_{I, k}\right)_{1 \leq I \leq N,-\mathbf{m} \leq k \leq \mathbf{m}}
$$

FSSL method is mesh-free and can be used with low approximation orders, since an order 2 or 3 generally gives a precision around order $10^{-2}$ in $\mathbb{H}^{1 / 2}$ of the single-layer densities, c.f. the convergence curve done for Experiment 1

\footnotetext{
${ }^{13}$ Our tests were run on the cluster Plafrim (www.plafrim.fr).

${ }^{14}$ This norm can be more 'pessimistic', i.e. a precision in norm $\mathbb{H}_{1 / 2}$ of the densities translates to a higher one in $L^{2}$ norm of the final solutions.
} 
180 $N \times(2 \times \mathbf{m}+1)$ for approximation order $\mathbf{m}$, are although dense ${ }^{15}$, but much smaller than those generated by Montjoie (for the same precision). For the latter method, assuming the mesh is fine enough to describe the obstacles, a good approximation order is between $\frac{5 \text { (mesh size) }}{\text { wavelength }}$ to $\frac{10 \text { (mesh size) }}{\text { wavelength }}$, called ' 5 -10 points per and order of the PML layer, which is not the case for FSSL. These factors keep the pre-processing time for FSSL drastically low compared to that for Montjoie.

In evaluating the diffracted field (46) for post-processing in FSSL, the most expensive ${ }^{16}$ operation is the computation of Hankel functions at distances between each evaluation point and each obstacle. The time cost increases with the definition of the visualization grid and the number of obstacles ${ }^{17}$. To diminish this drawback of FSSL, when the visualization grid is two-dimensional, we use Hermite cubic spline interpolation (with interpolation step size chosen so that the precision is $10^{-5}$ ), see description in Appendix B. Nevertheless, we will see in below experiments that FSSL even with exact evaluation is still faster due to its pre-processing time being drastically small.

We consider three numerical experiments, exploring the benefits of FSSL under the various factors (F.1-3),

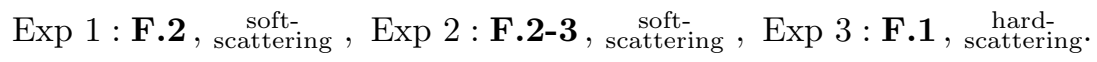

The experiments are done for obstacles with the same radius, equally spaced horizontally and vertically ${ }^{18}$, with the exception of Exp.1. We denote by $\mathrm{r}$ the obstacle radius, $d$ the distance between the centers of two adjacent obstacles, and 200 by $\lambda$ the wavelength of the plane wave. For the first two experiments, while the size of the visualization domains is within the acceptable range for Montjoi ${ }^{19}$ being $11 \lambda \times 16 \lambda$ and $31 \lambda \times 23 \lambda$ respectively, the obstacles are much smaller than the wavelength, being $\frac{1}{16} \lambda$ and $\frac{1}{21} \lambda$ respectively. The third experiment is designed to estimate the time cost of a 'forward problem', if FSSL or Montjoie is employed in a full-waveform inversion algorithm ${ }^{20}$ In this experiment, the obstacles are only $\frac{1}{6} \lambda$, but the domain is greater than $100 \lambda$, due to the required distance between the obstacles and the receivers (coming from physical experiments). While a lower time cost with FSSL is observed in all three experiments, it is in the third experiment that we see a spectacular gap between the two methods, with FSSL more than 2000 times faster than Montjoie, c.f. Table 3 This is due

\footnotetext{
${ }^{15} \mathrm{~A}$ property shared in general by methods in the boundary integral equation family.

${ }^{16}$ See Footnote 39

${ }^{17}$ See Footnote 40

18 However neither the code nor the method exploits these specificities (equal-sized obstacles and periodic spacing), and the same results should be observed for configurations with different sized obstacles as long as they are within the current smallness assumption (3).

${ }^{19}$ See Footnote 5 in Introduction.

20 The goal of inverse problem is to reconstruct the true parameters which give rise to the observed data. The 'forward problem' gives simulated data corresponding to a guessed set of parameters. In full waveform inversion (or quantitative inversion in general), one will need to solve the 'forward problem' a great number of times.
} 
to the fact that in the third experiment the visualization domain is quite big for Montjoie $(>100 \lambda)$, and secondly, the final solution is evaluated at 128 points rather than on a two-dimensional grid 21 .

Numerical experiment 1 . We consider the soft-scattering of $0^{\circ}$ plane wave (PW) with $\kappa=1.0$, by 6 very small obstacles on a domain of size $11 \lambda \times 16 \lambda$. The ratio between the obstacle radius $\mathrm{r}=0.4$, the distance between the centers of two adjacent obstacles $\mathrm{d}$ with $2 \leq \mathrm{d} \leq 2.9$, and the incident wavelength $\lambda \sim 6.3$ are

$$
\kappa \times \mathrm{r}=0.4 \quad, \quad \frac{\lambda}{\mathrm{r}} \sim 16 \quad, \quad 5 \leq \frac{\mathrm{d}}{\mathrm{r}} \leq 7 \quad, \quad 2 \leq \frac{\lambda}{\mathrm{d}} \leq 3 .
$$

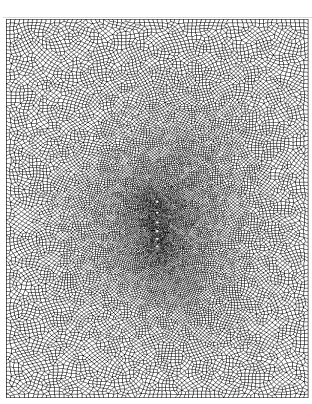

(a)

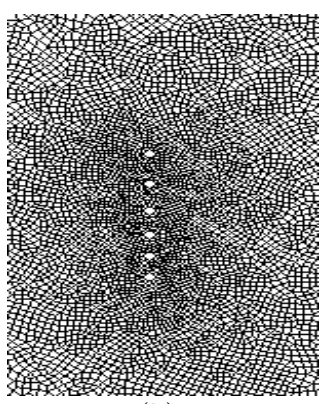

(b)

Figure 2: (a) MJ initial mesh with mesh size 0.679. (b) Mesh zoomed around obstacles. (Num. Exp. 1)

Comparison at precision $10^{-6}$ We choose MJ Q15 and FSSL 12 as reference solutions (at precision tolerance $10^{-12}$ ), c.f. Figure 3. Using the convergence curves in Figure 4, we compare between MJ Q5 and FSSL order 3, at precision $10^{-6}$. This choice is confirmed by their relative $L^{2}$ error compared to the reference solutions (shown below),

\begin{tabular}{c|c|c}
\multicolumn{2}{c|}{ Compare between } & Rel. $L^{2}$ error \\
\hline \hline FSSL 12 & FSSL 3 & $2.0 \times 10^{-6}$ \\
MJ Q15 & MJ Q5 & $4.18 \times 10^{-7}$ \\
\hline MJ Q3 & FSSL 3 & $1.98 \times 10^{-6}$
\end{tabular}

\begin{tabular}{|c|c|c|}
\hline \multicolumn{3}{|c|}{ Hermite interpolation precision is $10^{-6}$} \\
\hline \multicolumn{2}{|c|}{ Compare between } & Rel. $L^{2}$ error \\
\hline FSSL 3 Inter & $\overline{\text { FSSL } 3}$ & $3.92 \times 10^{-6}$ \\
\hline FSSL 3 In & MJ Q5 & $4.40 \times 10^{-}$ \\
\hline
\end{tabular}

From the comparison shown in Table 1 , at a precision of $10^{-6}$, FSSL using Hermite interpolation is more than 50 times faster than Montjoie.

Numerical Experiment 2. Here we also consider the soft-scattering by very small obstacles on a medium domain of size $31 \lambda \times 23 \lambda$, but with a much larger number of obstacles (200) than in Experiment 1. The specific ratios are

$$
\kappa \times \mathrm{r}=0.3, \frac{\lambda}{\mathrm{r}} \sim 21, \frac{\mathrm{d}}{\mathrm{r}} \sim 10, \frac{\lambda}{\mathrm{d}} \sim 2 .
$$

${ }^{21}$ This is the typical size (one dimensional rather than two) of simulated data in inversion. 


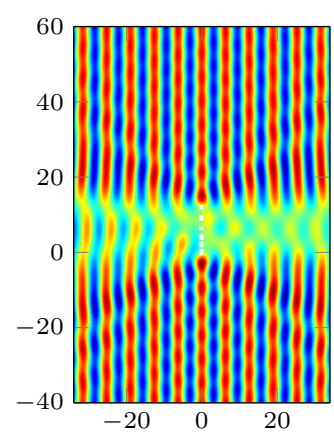

(a) MJ Q15

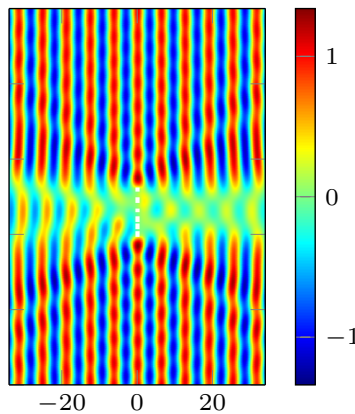

(b) FSSL 12.

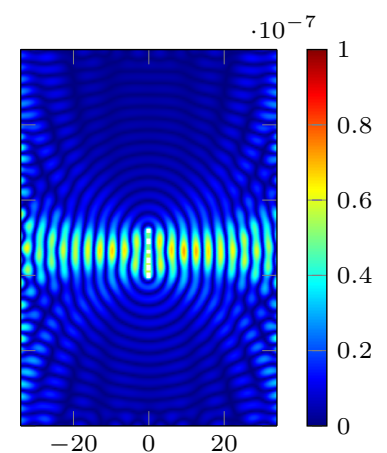

(c) Asbolute difference of real part. Relative $L^{2}$ error is $2.29 \times 10^{-8}$.

Figure 3: Real part of reference solution (total wave) in the soft-scattering of angle $0^{\circ}$ PW with $\lambda \sim 6.3$ by 6 obstacles with $\mathrm{r}=0.4$ and $2 \leq \mathrm{d} \leq 2.9$. (Num. Exp. 1)

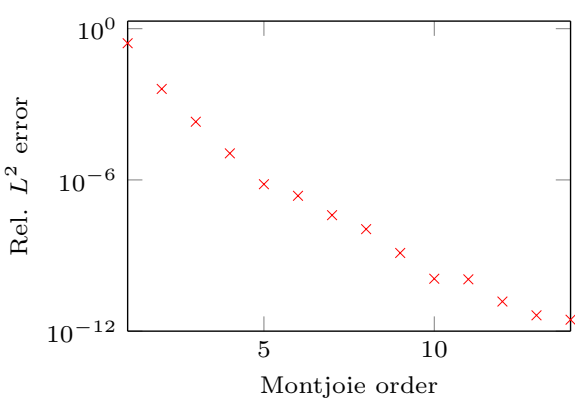

(a) Montjoie solution

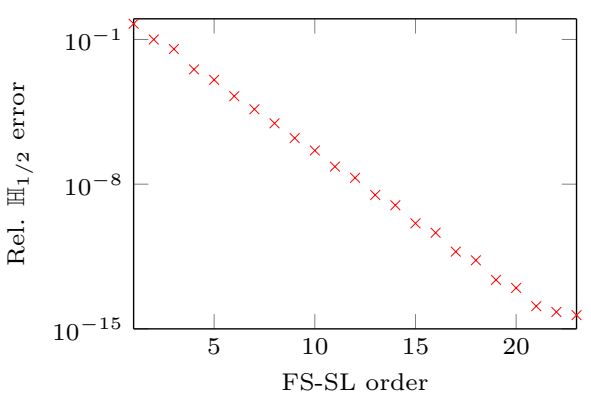

(b) FSSL density

Figure 4: Numerical Convergence using consecutive relative error (Num. Exp. 1).

\begin{tabular}{c|c|c}
$\begin{array}{c}\text { Pre-processing } \\
\text { by Mumps }\end{array}$ & $\begin{array}{c}\text { FSSL } \\
\text { Order 3 }\end{array}$ & $\begin{array}{c}\text { Montjoie } \\
\text { CG Q5 }\end{array}$ \\
\hline Size of lin. sys. & 42 & 435580 \\
\hline Task & \multicolumn{2}{|c}{ Time (s) } \\
\hline Construction & $1.08 \times 10^{-4}$ & 1.61 \\
Factorization & $6.95 \times 10^{-4}$ & 12.12 \\
Resolution & $1.03 \times 10^{-4}$ & 0.35 \\
\hline Total time & $1.45 \times 10^{-3}$ & 14.09
\end{tabular}

\begin{tabular}{l|c|c|c}
\multicolumn{3}{c}{ Evaluation on $\mathbf{4 0 0} \times \mathbf{4 0 0}$ grid } \\
& $\begin{array}{c}\text { Exact } \\
\text { eval }\end{array}$ & $\begin{array}{c}\text { Inter- } \\
\text {-polation }\end{array}$ & $\begin{array}{c}\text { MJ } \\
\text { Q5 }\end{array}$ \\
\hline \hline & 0.26 & 0.059 & 0.29 \\
\hline $\begin{array}{c}\text { Post-proc. } \\
\text { Pre-proc. }+\end{array}$ & 0.26 & 0.061 & 14.76
\end{tabular}

Table 1: Computational time comparison. At precision $10^{-6}$, FSSL using Hermite interpolation (0.06 s) takes 56 times less than MJ (14.7 s). (Num. Exp. 1) 
We choose Montjoie Q17 shown in Figure 5 and FSSL order 14 in Figure 6 as reference solutions at precision tolerance $10^{-8}$. The numerical convergence for both methods is shown in Figure 7. For 200 obstacles, it takes a lot of memory to obtain the convergence curve for Montjoi ${ }^{22}$.

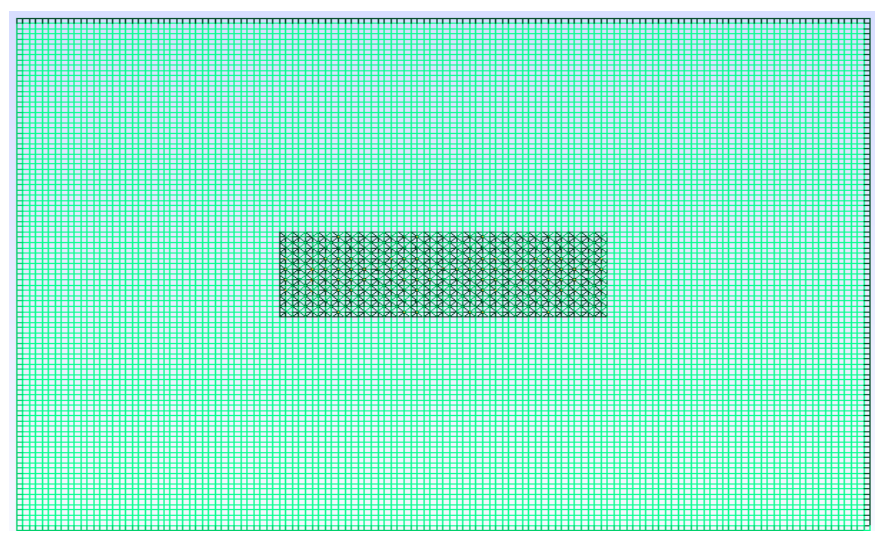

Figure 5: Initial MJ mesh with mesh size 0.13 (Num. Exp. 2)

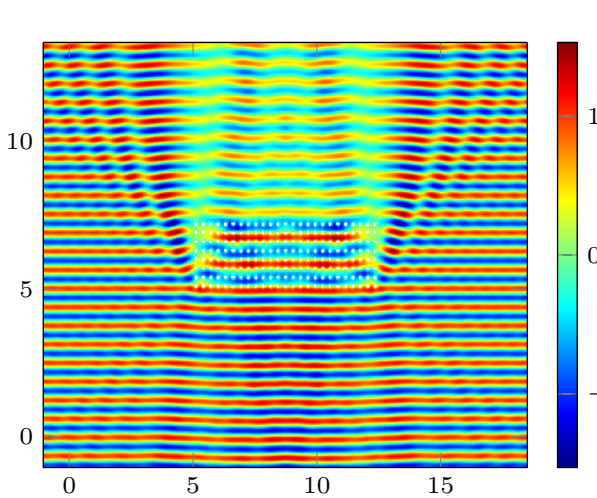

(a) Real part of FSSL Ref 14 total wave

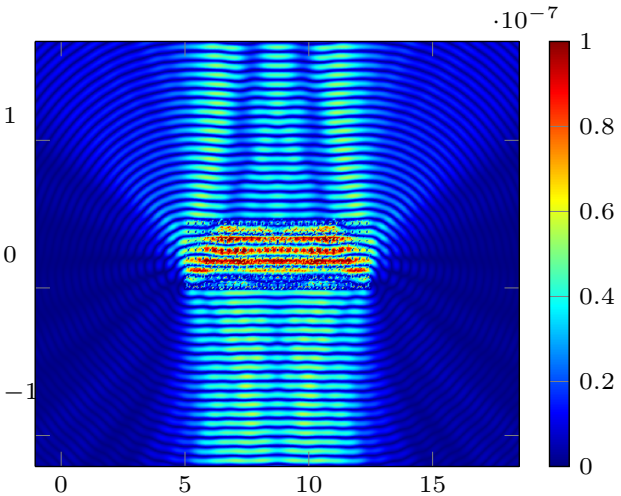

(b) Absolute difference of real part of total wave compared with MJ Q17. Rel. $L^{2}$ err.is $3.38 \times 10^{-8}$

Figure 6: Soft-scattering of PW of angle $90^{\circ}$ with $\kappa=10, \lambda \sim 0.63$ by 200 obstacles with $\mathrm{r}=0.03$ and $\mathrm{d}=0.3$. (Num. Exp. 2)

Comparison at precision $10^{-3}$ Using the convergence curves in Figure 4 , we compare between Montjoie Q6 and FSSL order 2. This choice is confirmed by

\footnotetext{
${ }^{22}$ In fact we stopped at consecutive precision $10^{-7}$. At order 16 , the overall simulation with Montjoie takes 21.39 GiB.
} 


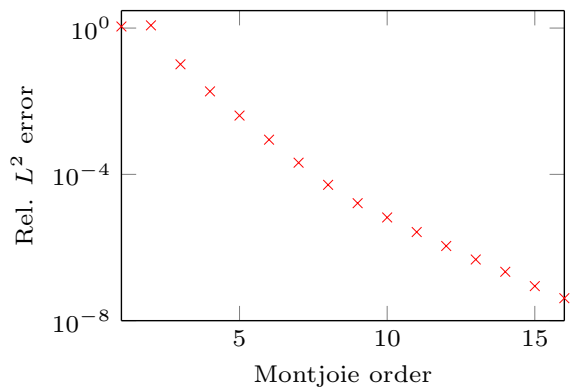

(a) Montjoie

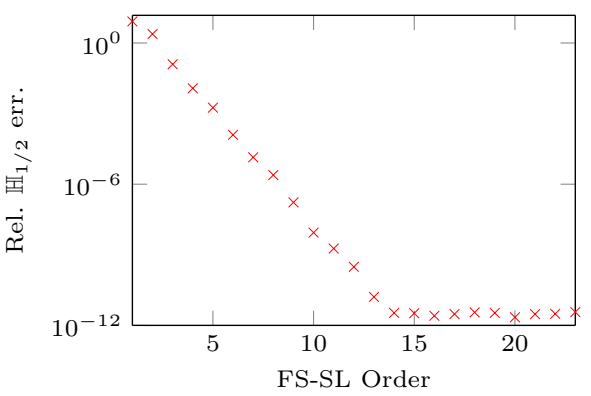

(b) FSSL (with Mumps)

Figure 7: Numerical Convergence using consecutive relative error (Num. Exp. 2).

\begin{tabular}{c|c|c}
$\begin{array}{c}\text { Pre-processing } \\
\text { by Mumps }\end{array}$ & $\begin{array}{c}\text { FSSL } \\
\text { Order 2 }\end{array}$ & $\begin{array}{c}\text { MJ } \\
\text { Q6 }\end{array}$ \\
\hline Size of lin. sys. & 1000 & 842677 \\
\hline \hline Task & \multicolumn{2}{|c}{ Time $(\mathrm{s})$} \\
\hline Construction & 0.055 & 1.97 \\
Factorization & 0.44 & 29.8 \\
Resolution & 0.003 & 0.35 \\
\hline Total time & $\mathbf{0 . 4 9 8}$ & $\mathbf{3 2 . 1 2}$
\end{tabular}

\begin{tabular}{c|c|c|c}
\multicolumn{4}{c}{ Evaluation on $\mathbf{4 0 0} \times \mathbf{4 0 0}$ grid } \\
& $\begin{array}{r}\text { Exact } \\
\text { eval }\end{array}$ & $\begin{array}{c}\text { Inter- } \\
\text {-polation }\end{array}$ & $\begin{array}{c}\text { MJ } \\
\text { Q6 }\end{array}$ \\
\hline \hline & 26.2 & 4.30 & 0.72 \\
\hline $\begin{array}{c}\text { Post-proc. } \\
\text { Post-proc. }\end{array}$ & $\mathbf{2 6 . 7 0}$ & $\mathbf{4 . 8 0}$ & $\mathbf{3 3 . 8 2}$
\end{tabular}

Table 2: Computational time comparison. At precision $10^{-3}$, FSSL using Hermite interpolation (4.8 s) takes 7 times less than MJ (33.8 s). (Num. Exp. 2)

their relative $L^{2}$ error compared to the reference solutions shown below,

\begin{tabular}{c|c|c}
\multicolumn{2}{c|}{ Compare between } & Rel. $L^{2}$ error \\
\hline FSSL 14 & FSSL 2 & $4.65 \times 10^{-5}$ \\
MJ Q17 & MJ Q6 & $6.52 \times 10^{-4}$ \\
MJ Q6 & FSSL2 & $6.84 \times 10^{-4}$ \\
\hline
\end{tabular}

\begin{tabular}{l|c|c} 
Hermite interpolation precision is $10^{-6}$ \\
\multicolumn{2}{c}{ Compare between } & Rel. $L^{2}$ error \\
\hline FSSL 2 Inter & FSSL 2 & $1.76 \times 10^{-5}$ \\
\hline FSSL 2 Inter & MJ Q6 & $6.85 \times 10^{-4}$
\end{tabular}

From the comparison shown in Table 2, we obtain the same conclusion as in Experiment 1. The cost of Hankel evaluation is quite large due to the high number of obstacles. However, with either kind of evaluation, FSSL still takes less time than Montjoie. In particular, for precision of $10^{-3}$, FSSL with Hermite interpolation takes 7 times less than Montjoie.

Numerical experiment 3. This experiment corresponds to a medium-sized physical experiment, in which acoustic vibration produced by an array of transducers - receivers ${ }^{23}$ is diffracted by 35 thin aluminum wires immersed in water, as shown in Figure 8. The transducer is placed at a required distance from the obstacles so that its signal ${ }^{24}$ can be approximated as a plane wave (of angle

\footnotetext{
${ }^{23}$ The transducer also acts as receiver.

${ }^{24}$ The central frequency of the input pulse is $500 \mathrm{kHz}$, and the speed of sound in water c $1478 \mathrm{~m} \mathrm{~s}^{-1}$. The corresponding wavenumber $\kappa$ is $2125.57 \mathrm{~m}^{-1}$ and spatial wavelength $\lambda$ is $2.96 \times 10^{-3} \mathrm{~m}$.
} 
$90^{\circ}$ ). The diffraction of this signal by thin wires is then approximated as the hard scattering of acoustic sound in fluid. The distance between the obstacles and the receivers results in a bigger domain (of interest) $117 \lambda \times 87 \lambda$. The ratios between the quantities in considerations are given by,

$$
\kappa \times \mathrm{r} \sim 1.1 \quad, \quad \frac{\mathrm{d}}{\mathrm{r}} \sim(23,19) \quad, \quad \frac{\lambda}{\mathrm{r}} \sim 5.912 \quad, \quad \frac{\lambda}{\mathrm{d}} \sim 0.3 .
$$

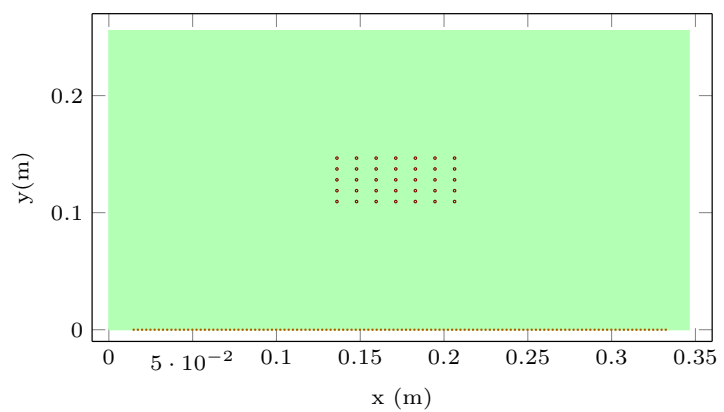

Figure 8: Obstacle and Receptor configurations. The 35 obstacles of radius $0.5 \mathrm{~mm}$ are spaced horizontally by $11.7 \mathrm{~mm}$ and vertically by $9.28 \mathrm{~mm}$ and are immersed in water. The 128 receptors are equally spaced by $2.5 \mathrm{~mm}$. (Num. Exp 3)

225 Comparison at precision $10^{-4}$ Regarding the value of the diffracted wave at 128 receptors, for FSSL (with Lapack), the relative $L^{2}$ error between order 12 and order 4 is $2.82 \times 10^{-6}$, for Montjoie, the relative $L^{2}$ error between Q12 and Q8 with one time mesh refinement ${ }^{25}$, called MJ Q8 Ref 2 , is $1.42 \times 10^{-4}$. Thus for a precision of order $10^{-4}$, we can choose FSSL order 4 and MJ Q8 Ref2, 230 whose relative $L^{2}$ error is $1.4808 \times 10^{-4}$, c.f. Figure 9. From the comparison shown in Table 3, at a precision $10^{-4}$, FSSL with exact post-processing $(0.003$ s) is more than 2000 faster than Montjoie (61.4 s).

\begin{tabular}{c|c|c|c|c} 
& Size of LS & Pre-proc. Time & $\begin{array}{c}\text { Post-proc. } \\
\text { at 128 receivers }\end{array}$ & Total time \\
\hline \hline FSSL 4 & 315 & $2.40 \times 10^{-2}$ & $6.58 \times 10^{-3}$ & $3.06 \times 10^{-2}$ \\
\hline MJ Q8 Ref 2 & 993870 & 61.27 & 0.13 & 61.4
\end{tabular}

Table 3: Computational time comparison. At precision $10^{-4}$, FSSL with exact postprocessing (0.003 s) is 2046 times faster than MJ (61.4 s). (Num. Exp. 3)

Remark 6. If we collect the data of the above experiment on a $400 \times 400$ grid, then the post-processing time for MJ is 1.16s, for FSSL with exact evaluation 7.59s, and with Hermite interpolation 2.34s. As a result, the total time

\footnotetext{
${ }^{25}$ In order to improve the precision of MJ, we can increase the order of approximation, or refine the mesh. The latter is generally less costly.
} 


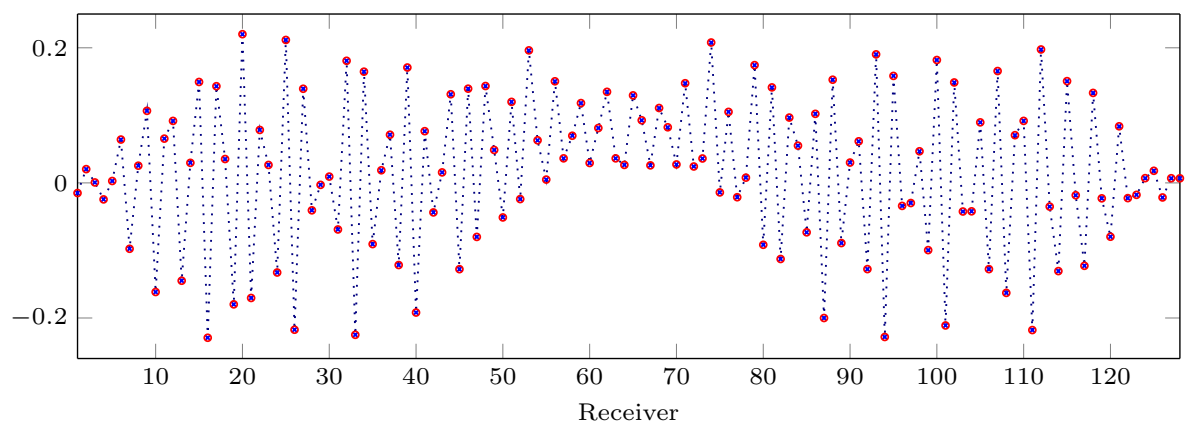

Figure 9: Real part of hard-diffracted wave at 128 receptors for FSSL $4 \cdots \cdots \cdots$ and MJ Q8 Rafo Rel $L^{2}$ error is $1.48 \times 10^{-4}$. (Num. Exp. 3)

cost for MJ Q8 Raf 2 is 62.43s, for FSSL with exact evaluation 7.63s (thus 8 times faster), and with interpolation Hermite interpolation $2.39 \mathrm{~s}$ (thus 26 times faster).

\section{Solvers performance comparison within FSSL codes}

As motivated in the introduction, we need to address the questions, when using FSSL, whether a direct or an iterative solver is better, and within the iterative family which kind of preconditioner will give the fastest convergence. We will see that different formations of obstacles require different solvers to obtain optimality or even just convergence (for the iterative ones).

The numerical comparisons between direct solvers (Mumps, Lapack and Scalapack) and iterative solvers in the family of GMRES with restart are carried out for closely-spaced obstacles 26 , characterized by the ratios $\frac{\mathrm{d}}{\mathrm{r}}=10$ and $\frac{\lambda}{\mathrm{d}}=$ 2 , and for far-apart obstacles by $\frac{\mathrm{d}}{\mathrm{r}}=200, \frac{\lambda}{\mathrm{d}}=0.3$. The random configuration in [6, 21] corresponds more to our 'closely-spaced' experiments 27 . For both types of configuration, we will assume soft-scattering, however the same result should be expected for hard-scattering.

The comparison is done for preconditioners associated to Jacobi and GaussSeidel and for each of them we consider a left or right conditioning position. For brevity, we add $L_{-}$or $R_{-}$to the name of the preconditioners to indicate this. Each

\footnotetext{
${ }^{26}$ As in previous sections, we assume that the obstacles are of the same radius, equally spaced horizontally and vertically. We have denoted by $\mathrm{r}$ the obstacle radius, $\mathrm{d}$ the distance between the centers of two adjacent obstacles, and by $\lambda$ the wavelength of the plane wave. As noted before, neither the code nor the method makes use of these assumptions (equal radius, equal spacing

${ }^{27}$ Intuitively, the configuration of equally and closely spaced obstacles should give more illconditioned multi-scattering matrix than in a randomly spaced one, because of the enhanced interaction of the obstacles.
} 
inversion with GMRES with restart comes with parameters: precision tolerance, maximum number of iterations, and Krylov space size (also called restart). We refer readers to [28, Appendix C] and [20] and the references cited therein for a more detailed discussion of the method. Intuitively, a preconditioner $\mathcal{P}$ to a linear equation with coefficient $A$, is defined such that its inverse $\mathcal{P}^{-1}$ approximates $A^{-1}$. Denote by $L, D, U$ the strictly lower, the diagonal and the strictly upper part of matrix $A$. We write

$$
M_{u}=U+D, N_{u}=-L ; M_{l}=L+D, N_{l}=-U ; R=-L-U .
$$

As a result, we have the following splitings of $A$,

$$
A=L+D+U=M_{u}-N_{u}=M_{l}-N_{l}=D-R .
$$

1. The backward Gauss-Seidel (BGS) preconditioner is $\mathcal{P}=M_{u}$.

2. The forward Gauss-Seidel (FGS) preconditioner is $\mathcal{P}=M_{l}$.

3. The Jacobi preconditioner is $\mathcal{P}=D$.

4. The 2nd-order Jacobi (2Jacobi) preconditioner is defined as

$$
\mathcal{P}^{-1}=D^{-1}(R+D) D^{-1} .
$$

The action of the inverse can also be described as applying two Jacobi iterations; in particular, $u=\mathcal{P}^{-1} f$ is the solution ${ }^{28}$ to

$$
D \tilde{u}=f ; D u=R \tilde{u}+f .
$$

The above operator can be formally seen as a 'second-order' approximation of the Neumann series of $A^{-1}$ via the splitting ${ }^{29} A=D-R$.

5. The 2nd-order Forward Gauss-Seidel (2FGS) preconditioner is

$$
\mathcal{P}^{-1}=M_{l}^{-1}\left(N_{l}+M_{l}\right) M_{l}^{-1} .
$$

The action of its inverse is such that $u=\mathcal{P}^{-1} f$ is the solution 30 to

$$
\text { Forward GS : } M_{l} \tilde{u}=f \text {; Forward GS : } M_{l} u=N_{l} \tilde{u}+f \text {. }
$$

Similarly to 2Jacobi, the above operator can be seen as a 'second-order' approximation of the Neumann series of $A^{-1}$ via the splitting $A=M_{l}-N_{l}$.

6. The Symmetric Gauss-Seidel (SGS) preconditioner is defined as

$$
\mathcal{P}=M_{u} D^{-1} M_{l} \quad \text { (Symmetric GS preconditioner) }
$$

The action of its inverse is such that $u=\mathcal{P}^{-1} f$ is the solution ${ }^{31}$ to

$$
\text { Backward GS : } M_{u} \tilde{u}=f \text {; Forward GS : } M_{l} u=N_{l} \tilde{u}+f \text {. }
$$

\footnotetext{
${ }^{28}$ This is seen as $u=\mathcal{P}^{-1} f=D^{-1}(R+D) D^{-1} f$.

${ }^{29}$ This is seen as $A=D-R=D\left(\operatorname{Id}-D^{-1} R\right) \Rightarrow A^{-1}=\left(\operatorname{Id}-D^{-1} R\right)^{-1} D^{-1}=D^{-1}+$ $D^{-1} R D^{-1}+\sum_{k=2}^{\infty}\left(D^{-1} R\right)^{k} D^{-1}$.

${ }^{30}$ This is seen as $u=\mathcal{P}^{-1} f=M_{l}^{-1}\left(N_{l}+M_{l}\right) M_{l}^{-1} f$.

${ }^{31}$ This is seen as $u=M_{l}^{-1} N_{l} M_{u}^{-1} f+M_{l}^{-1} f=M_{l}^{-1}\left(N_{l}+M_{u}\right) M_{u}^{-1} f=M_{l}^{-1} D M_{u}^{-1} f$.
} 


\section{The Lower-Upper Symmetric Gauss-Seidel (LUSGS) preconditioner} is

$$
\mathcal{P}=M_{l} D^{-1} M_{u} \quad \text { (LU SGS Preconditioner) . }
$$

The action of its inverse is such that $u=\mathcal{P}^{-1} f$ is the solution 32 to

$$
\text { Forward GS : } \quad M_{l} \tilde{u}=f \text {; Backward GS : } \quad M_{u} u=N_{u} \tilde{u}+f .
$$

The seven preconditioners in our study include two that are mentioned in [6, 21]: the block Jacobi and one which is comparable ${ }^{33}$ to our 2nd-order Jacobi. We incorporate the GMRES (with restart) library [20] in our Fortran codes and use its feature which allows users to add routines to define multiplication by coefficient matrices, preconditioners and matrix vector multiplication.

Numerical Experiment 4. To have a feel for the convergence of the preconditioners in closely-spaced configurations, we first start with a reasonable number of obstacles (200 in Exp. 4a), then increase to a very large number of them (1616 in Exp. 4b) in order to see how their convergence changes. This process selects the most robust preconditioners. These comparisons are carried out in sequential execution ${ }^{34}$. In the last experiment of this group (Exp. 4c), a more 'practical' comparison is done, taking into consideration the possibility of parallelization of the direct family, to give a realistic user's time cost for simulating very large configurations (2000 obstacles). The results of Exp 4a and $4 \mathrm{~b}$ are listed in Table 4, 4c in Table 5. The ratios of the configuration are

$$
\kappa \times \mathrm{r}=0.3 \quad, \quad \frac{\lambda}{\mathrm{r}} \sim 21 \quad, \quad \frac{\mathrm{d}}{\mathrm{r}}=10 \quad, \quad \frac{\lambda}{\mathrm{d}} \sim 2 .
$$

Note that $\kappa \times \mathrm{r}<2$ hence we are in the invertible region.

For 200 obstacles, we obtain convergence for all listed choices with or without preconditioning. In general, the direct solvers take less time than the iterative ones, with the exception of the SGS and LUSGS family which are as fast as Mumps. The iterative solvers give lower precision density (with precision $10^{-3}$ ) compared to that by direct solvers (at precision $10^{-12}$ ). The performance of the 270 GMRES solvers falls into 4 distinct groups, with big performance gaps between them: no pre-conditioning takes 820 iterations, (left and right) preconditioning with Jacobi and 2Jacobi taking $\sim 500$ iterations, (left and right) preconditioning BGS, FGS, and 2FGS takes $~ 199$ iterations, the most efficient group being with SGS and LUSGS which takes $\sim 76$ iterations. The Jacobi family (Jacobi and 2Jacobi), containing only the information of diagonal blocks, does not perform as well as Gauss-Seidel ones, the latter containing informations of off-diagonal blocks. The first group has difficulty converging, even for 200 obstacles, and does not converge for 1616 obstacles. Intuitively, with the strong interaction

\footnotetext{
${ }^{32}$ This is seen as $u=M_{u}^{-1} N_{u} \tilde{u}+M_{u}^{-1} f=M_{u}^{-1}\left(N_{u}+M_{l}\right) M_{l}^{-1} f=M_{u}^{-1} D M_{l}^{-1} f$.

${ }^{33}$ However, we did not use a sparsified version of the preconditioner as is done in 66 21.

${ }^{34}$ This is a 'fairer' comparison (than Exp. 4c) since the current GMRES codes can only be run in sequential.
} 
among closely-spaced obstacles, the coefficient matrix ceases to be diagonally dominant, with the off-diagonal blocks (describing interaction between different obstacles) comparable in size to the diagonal ones (describing self-interaction). This confirms the need for preconditioning of the multi-scattering matrix.

For the case of 1616 obstacles, we only obtain convergence for SGS and LUSGS, confirming the fact that this group is the most appropriate choice of 285 preconditioner for a multiple-scattering setting. However, with a large number of obstacles, the number of iterations needed for GMRES to converge increases drastically, taking $\sim 880$ iterations (compared with only $\sim 76$ for 200 obstacles). As a result, the direct solvers are much more efficient than the GMRES ones, with Lapack being the optimal choice. Mumps while not adapted for dense matrices is still faster compared to the GMRES solvers in this case.

\begin{tabular}{|c|c|c|c|c|c|c|c|c|}
\hline & \multicolumn{4}{|c|}{ Case 200 obstacles } & \multicolumn{4}{|c|}{ Case 1616 obstacles } \\
\hline $\begin{array}{l}\text { Name } \\
\text { Method }\end{array}$ & $\mathrm{Cv}$ & $\begin{array}{c}\boldsymbol{\delta}_{\text {err }} \text { in } \\
\mathbb{H}_{1 / \mathbf{2}}\end{array}$ & $\begin{array}{c}\sharp \\
\text { Iter }\end{array}$ & $\begin{array}{c}\text { Time } \\
(\mathrm{s})\end{array}$ & $\mathrm{Cv}$ & $\begin{array}{c}\boldsymbol{\delta}_{\text {err }} \text { in } \\
\mathbb{H}_{1 / 2}\end{array}$ & $\begin{array}{c}\sharp \\
\text { Iter }\end{array}$ & $\underset{(\mathrm{s})}{\text { Time }}$ \\
\hline Mumps & $\mathrm{n} / \mathrm{a}$ & 0 & $\mathrm{n} / \mathrm{a}$ & 0.5 & $\mathrm{n} / \mathrm{a}$ & 0 & $\mathrm{n} / \mathrm{a}$ & 130 \\
\hline \multirow[t]{2}{*}{ Lapack } & $\mathrm{n} / \mathrm{a}$ & $10^{-12}$ & $\mathrm{n} / \mathrm{a}$ & 0.1 & $\mathrm{n} / \mathrm{a}$ & $10^{-10}$ & $\mathrm{n} / \mathrm{a}$ & 42.7 \\
\hline & \multicolumn{4}{|c|}{$\begin{array}{l}\text { GMRES parameters } \\
\left(10^{-6}, 2000,100\right)\end{array}$} & \multicolumn{4}{|c|}{$\begin{array}{c}\text { GMRES parameters } \\
\left(10^{-6}, 2000,150\right)\end{array}$} \\
\hline NoPreCond & $\bar{Y}$ & $5 \times 10^{-3}$ & 820 & 0.9 & $\mathrm{~N}$ & $\mathrm{n} / \mathrm{a}$ & $\mathrm{n} / \mathrm{a}$ & $\mathrm{n} / \mathrm{a}$ \\
\hline L_Jacobi & $\mathrm{Y}$ & $5 \times 10^{-3}$ & 656 & 0.8 & $\mathrm{~N}$ & $\mathrm{n} / \mathrm{a}$ & $\mathrm{n} / \mathrm{a}$ & $\mathrm{n} / \mathrm{a}$ \\
\hline L_FGS & $\bar{Y}$ & $2 \times 10^{-3}$ & 239 & 0.5 & $\mathrm{~N}$ & $\mathrm{n} / \mathrm{a}$ & $\mathrm{n} / \mathrm{a}$ & $\mathrm{n} / \mathrm{a}$ \\
\hline L_BGS & $\mathrm{Y}$ & $4 \times 10^{-3}$ & 197 & 0.4 & $\mathrm{~N}$ & $\mathrm{n} / \mathrm{a}$ & $\mathrm{n} / \mathrm{a}$ & $\mathrm{n} / \mathrm{a}$ \\
\hline L_2Jacob & $\mathrm{Y}$ & $5 \times 10^{-3}$ & 594 & 2.21 & $\mathrm{~N}$ & $\mathrm{n} / \mathrm{a}$ & $\mathrm{n} / \mathrm{a}$ & $\mathrm{n} / \mathrm{a}$ \\
\hline L_2FGS & $\mathrm{Y}$ & $1 \times 10^{-3}$ & 169 & 1.0 & $\mathrm{~N}$ & $\mathrm{n} / \mathrm{a}$ & $\mathrm{n} / \mathrm{a}$ & $\mathrm{n} / \mathrm{a}$ \\
\hline L_SGS & $\bar{Y}$ & $2 \times 10^{-3}$ & 76 & 0.3 & $\bar{Y}$ & $4 \times 10^{-1}$ & 757 & 274 \\
\hline L_LUSGS & $\mathrm{Y}$ & $1 \times 10^{-3}$ & 77 & 0.3 & $\mathrm{Y}$ & $1 \times 10^{-1}$ & 897 & 325 \\
\hline R_Jacobi & $\bar{Y}$ & $4 \times 10^{-3}$ & 660 & 1.05 & $\mathrm{~N}$ & $\mathrm{n} / \mathrm{a}$ & $\mathrm{n} / \mathrm{a}$ & $\mathrm{n} / \mathrm{a}$ \\
\hline R_FGS & $\mathrm{Y}$ & $3 \times 10^{-3}$ & 199 & 0.5 & $\mathrm{~N}$ & $\mathrm{n} / \mathrm{a}$ & $\mathrm{n} / \mathrm{a}$ & $\mathrm{n} / \mathrm{a}$ \\
\hline R_BGS & $\bar{Y}$ & $3 \times 10^{-3}$ & 198 & 0.4 & $\mathrm{~N}$ & $\mathrm{n} / \mathrm{a}$ & $\mathrm{n} / \mathrm{a}$ & $\mathrm{n} / \mathrm{a}$ \\
\hline R_2Jaco & $\mathrm{Y}$ & $4 \times 10^{-3}$ & 600 & 1.70 & $\mathrm{~N}$ & $\mathrm{n} / \mathrm{a}$ & $\mathrm{n} / \mathrm{a}$ & $\mathrm{n} / \mathrm{a}$ \\
\hline R_2FGS & $\mathrm{Y}$ & $3 \times 10^{-3}$ & 155 & 0.9 & $\mathrm{~N}$ & $\mathrm{n} / \mathrm{a}$ & $\mathrm{n} / \mathrm{a}$ & $\mathrm{n} / \mathrm{a}$ \\
\hline R_SGS & $\bar{Y}$ & $3 \times 10^{-3}$ & 75 & 0.3 & $\bar{Y}$ & $2 \times 10^{-1}$ & 886 & 321 \\
\hline R_LUSGS & $\mathrm{Y}$ & $3 \times 10^{-3}$ & 74 & 0.3 & Y & $2 \times 10^{-1}$ & 897 & 325 \\
\hline
\end{tabular}

Table 4: Soft-scattering of PW with $90.0^{\circ}$ and $\kappa=10$ by obstacles with $\mathrm{r}=0.03$ and $\mathrm{d}=0.3$. FSSL method order $=2$. ' $\mathrm{Cv}$ ' $=$ Convergence. For full results, see also 28 , page 42, 44] (Numerical result $\mathbf{4 a - 4 b )}$

For 2000 obstacles, we limit the comparison to the robust Lower-Upper Symmetric Gauss-Seidel (LUSGS) and Symmetric Gauss-Seidel (SGS) preconditioners and the direct solvers. For this experiment, we add a comparison in $L^{2}$ norm of the final field, since the relative error in $\mathbb{H}_{1 / 2}$ can be pessimistic; secondly, the comparison is done in parallel execution and with solver Scalapack. Due to the denseness of the matrix, parallelization does not improve the execution 
time for Mumps. On the other hand, the time gain obtained with Lapack is further increased by its parallel version Scalapack. In order to simulate multiplescattering by 2000 small and closely-spaced obstacles, the optimal choice is using Scalapack and Hermite interpolation, which takes a total of 1 min and 10 secs for a visualization of the solution on a $800 \times 800$ grid. The results are listed in Table 5

\begin{tabular}{|c|c|c|c|c|c|c|c|}
\hline Solver & $\begin{array}{l}\text { Post- } \\
\text { proc } \\
(\mathrm{n} 16)\end{array}$ & $\begin{array}{c}\text { Rel } \\
\mathbb{H}_{1 / 2} \\
\text { diff }\end{array}$ & $\begin{array}{l}\operatorname{Rel} L^{2} \\
\quad \operatorname{diff}\end{array}$ & $\begin{array}{c}\sharp \\
\text { iter }\end{array}$ & $\begin{array}{l}\text { Pre- } \\
\text { proc. } \\
\text { time } \\
(\mathrm{s})\end{array}$ & $\begin{array}{l}\text { Post- } \\
\text { proc. } \\
\text { time } \\
(\mathrm{s})\end{array}$ & $\begin{array}{l}\text { Total } \\
(\mathrm{s})\end{array}$ \\
\hline $\begin{array}{l}\text { Mumps (n16) } \\
\text { Mumps (n16) }\end{array}$ & $\begin{array}{l}\text { Exact } \\
\text { Inter }\end{array}$ & $\begin{array}{l}3 \times 10^{-10} \\
3 \times 10^{-10}\end{array}$ & $\begin{array}{l}8 \times 10^{-14} \\
9 \times 10^{-6} \\
\end{array}$ & $\begin{array}{l}\mathrm{n} / \mathrm{a} \\
\mathrm{n} / \mathrm{a}\end{array}$ & $\begin{array}{l}242 \\
242 \\
\end{array}$ & $\begin{array}{l}96.0 \\
36.0 \\
\end{array}$ & $\begin{array}{l}338 \\
278 \\
\end{array}$ \\
\hline $\begin{array}{l}\text { Lapack (n1) } \\
\text { Lapack (n1) }\end{array}$ & $\begin{array}{l}\text { Exact } \\
\text { Inter }\end{array}$ & $\begin{array}{l}0 \\
0\end{array}$ & $\begin{array}{c}0 \\
9 \times 10^{-6} \\
\end{array}$ & $\begin{array}{l}\mathrm{n} / \mathrm{a} \\
\mathrm{n} / \mathrm{a}\end{array}$ & $\begin{array}{l}80.4 \\
80.4 \\
\end{array}$ & $\begin{array}{l}96.0 \\
37.5 \\
\end{array}$ & $\begin{array}{l}176 \\
118 \\
\end{array}$ \\
\hline $\begin{array}{l}\text { R_LUSGS (n1) } \\
\text { R_LUSGS (n1) }\end{array}$ & $\begin{array}{l}\text { Exact } \\
\text { Inter }\end{array}$ & $\begin{array}{l}1 \times 10^{-1} \\
1 \times 10^{-1}\end{array}$ & $\begin{array}{l}4 \times 10^{-5} \\
4 \times 10^{-5}\end{array}$ & $\begin{array}{l}1146 \\
1146\end{array}$ & $\begin{array}{l}573 \\
573 \\
\end{array}$ & $\begin{array}{l}95.8 \\
36.2 \\
\end{array}$ & $\begin{array}{l}669 \\
609 \\
\end{array}$ \\
\hline $\begin{array}{l}\text { R_SGS (n1) } \\
\text { R_SGS (n1) }\end{array}$ & $\begin{array}{l}\text { Exact } \\
\text { Inter }\end{array}$ & $\begin{array}{l}1 \times 10^{-1} \\
1 \times 10^{-1}\end{array}$ & $\begin{array}{l}4 \times 10^{-5} \\
4 \times 10^{-5}\end{array}$ & $\begin{array}{l}1151 \\
1151\end{array}$ & $\begin{array}{l}598 \\
598 \\
\end{array}$ & $\begin{array}{l}95.8 \\
36.2 \\
\end{array}$ & $\begin{array}{l}694 \\
635 \\
\end{array}$ \\
\hline $\begin{array}{l}\text { Scala (n16) } \\
\text { Scala (n16) }\end{array}$ & $\begin{array}{l}\text { Exact } \\
\text { Inter }\end{array}$ & $\begin{array}{l}3 \times 10^{-10} \\
3 \times 10^{-10}\end{array}$ & $\begin{array}{l}8 \times 10^{-14} \\
9 \times 10^{-6}\end{array}$ & $\begin{array}{l}\mathrm{n} / \mathrm{a} \\
\mathrm{n} / \mathrm{a}\end{array}$ & $\begin{array}{l}34.6 \\
34.6\end{array}$ & $\begin{array}{l}95.6 \\
36.1\end{array}$ & $\begin{array}{r}130 \\
70.9\end{array}$ \\
\hline
\end{tabular}

Table 5: Soft-scattering of PW with $90^{\circ}$ and $\kappa=10.0$ by 2000 obstacles with $\mathrm{r}=0.03$, and $\mathrm{d}=0.30$. FS-SL method order $=2$. Matrix size $=10^{4} \times 10^{4}$. For parallel execution, the number after $-n$ indicates the number of processors. GMRES parameters $\left(10^{-6}\right.$, 5000, 400). For full results, see also [28, page 49] (Num. Exp. 4c)

Numerical Result 5. With incident PW of the same wavelength and same number (2000) obstacles as in Exp. 4c, we now consider a configuration in which the obstacles are further apart. The specific ratios are

$$
\kappa \times \mathrm{r}=0.1 \quad, \quad \frac{\lambda}{\mathrm{r}} \sim 63 \quad, \quad \frac{\mathrm{d}}{\mathrm{r}}=200 \quad, \quad \frac{\lambda}{\mathrm{d}} \sim 0.3 .
$$

The number of iterations for GMRES drastically drop, taking only 56 iterations. This is the same number of iterations needed for 200 closely-spaced obstacles. In fact, GMRES with LUSGS preconditioner is twice as fast as Lapack, and is almost comparable in performance to Scalapack running on 16 processors. On the other hand, the distance between obstacles has small impact on the direct solvers. Their performance time stays approximately the same. As a result, for the case where the obstacles are far-way, under low precision, one has the option 310 of using GMRES with either LUSGS or SGS as preconditioners. The results are listed in Table 6 . 


\begin{tabular}{|c|c|c|c|c|c|c|c|}
\hline Solver & $\begin{array}{c}\text { Post- } \\
\text { proc } \\
(\mathrm{n} 16)\end{array}$ & $\begin{array}{c}\text { Rel } \\
\mathbb{H}_{1 / 2} \\
\text { diff }\end{array}$ & $\begin{array}{l}\operatorname{Rel} L^{2} \\
\quad \operatorname{diff}\end{array}$ & $\stackrel{\sharp}{\text { iter }}$ & $\begin{array}{l}\text { Pre- } \\
\text { proc. } \\
\text { time } \\
(\mathrm{s})\end{array}$ & $\begin{array}{l}\text { Post- } \\
\text { proc. } \\
\text { time } \\
(\mathrm{s})\end{array}$ & $\begin{array}{l}\text { Total } \\
(\mathrm{s})\end{array}$ \\
\hline $\begin{array}{l}\text { Mumps (n1) } \\
\text { Mumps (n1) }\end{array}$ & $\begin{array}{l}\text { Exact } \\
\text { Inter }\end{array}$ & $\begin{array}{l}0.0 \\
0.0 \\
\end{array}$ & $\begin{array}{c}0.0 \\
1 \times 10^{-5} \\
\end{array}$ & $\begin{array}{l}\mathrm{n} / \mathrm{a} \\
\mathrm{n} / \mathrm{a}\end{array}$ & $\begin{array}{l}251 \\
251 \\
\end{array}$ & $\begin{array}{l}96.0 \\
37.5 \\
\end{array}$ & $\begin{array}{l}347 \\
289 \\
\end{array}$ \\
\hline $\begin{array}{l}\text { Lapack (n1) } \\
\text { Lapack (n1) }\end{array}$ & $\begin{array}{l}\text { Exact } \\
\text { Inter }\end{array}$ & $\begin{array}{l}4 \times 10^{-12} \\
4 \times 10^{-12}\end{array}$ & $\begin{array}{l}2 \times 10^{-15} \\
1 \times 10^{-5}\end{array}$ & $\begin{array}{l}\mathrm{n} / \mathrm{a} \\
\mathrm{n} / \mathrm{a}\end{array}$ & $\begin{array}{l}79.9 \\
79.9 \\
\end{array}$ & $\begin{array}{l}96.0 \\
37.5 \\
\end{array}$ & $\begin{array}{l}176 \\
118 \\
\end{array}$ \\
\hline $\begin{array}{l}\text { R_LUSGS (n1) } \\
\text { R_LUSGS (n1) }\end{array}$ & $\begin{array}{l}\text { Exact } \\
\text { Inter }\end{array}$ & $\begin{array}{l}3 \times 10^{-4} \\
3 \times 10^{-4}\end{array}$ & $\begin{array}{l}1 \times 10^{-7} \\
1 \times 10^{-5} \\
\end{array}$ & $\begin{array}{l}57 \\
57 \\
\end{array}$ & $\begin{array}{l}37.5 \\
37.5 \\
\end{array}$ & $\begin{array}{l}96.0 \\
37.5 \\
\end{array}$ & $\begin{array}{c}134 \\
75.3 \\
\end{array}$ \\
\hline $\begin{array}{l}\text { R_SGS (n1) } \\
\text { R_SGS (n1) }\end{array}$ & $\begin{array}{l}\text { Exact } \\
\text { Inter }\end{array}$ & $\begin{array}{l}4 \times 10^{-4} \\
4 \times 10^{-4}\end{array}$ & $\begin{array}{l}1 \times 10^{-7} \\
1 \times 10^{-5} \\
\end{array}$ & $\begin{array}{l}56 \\
56 \\
\end{array}$ & $\begin{array}{l}37.0 \\
37.0 \\
\end{array}$ & $\begin{array}{l}96.0 \\
37.5 \\
\end{array}$ & $\begin{array}{l}133 \\
74.6 \\
\end{array}$ \\
\hline $\begin{array}{l}\text { Scala (n16) } \\
\text { Scala (n16) }\end{array}$ & $\begin{array}{l}\text { Exact } \\
\text { Inter }\end{array}$ & $\begin{array}{l}1 \times 10^{-11} \\
1 \times 10^{-11}\end{array}$ & $\begin{array}{l}4 \times 10^{-15} \\
1 \times 10^{-5}\end{array}$ & $\begin{array}{l}\mathrm{n} / \mathrm{a} \\
\mathrm{n} / \mathrm{a}\end{array}$ & $\begin{array}{l}34.9 \\
34.9\end{array}$ & $\begin{array}{l}96.0 \\
37.5\end{array}$ & $\begin{array}{l}131 \\
72.5\end{array}$ \\
\hline
\end{tabular}

Table 6: Soft-scattering of PW with $90.0^{\circ}$ and $\kappa=10.0$ by 2000 obstacles with $\mathrm{r}=0.01$, and $\mathrm{d}=2.00$. FSSL method order 2. Matrix size $=10000 \times 10000$. GMRES Solver $\left(10^{-7}, 5000,500\right)$. For parallel execution, the number after $-n$ indicates the number of processors. For full results, see also [28, page 51] (Num. Exp. 5)

Numerical Result $\boldsymbol{6}$. The only solver that can handle very large number of small obstacles is currently the robust direct solver Scalapack. With the same ratios, from Experiment 4, for the soft-scattering of 10000 small closely-spaced obstacles, FSSL with Scalapack takes 24 mins 40 secs on 48 processors, see Figure 10. Most of this cost is pre-processing, 22 mins 20 secs.

Remark 7. As noted from experiment 5 that the distance between the obstacles has small impact on the performance of direct solvers, we should expect the same time cost, if these obstacles are far-apart (e.g. with the ratios of Exp 5). In addition, the same results should be expected for hard-scattering.

Observations from the solver comparisons. For closely-spaced configuration and FSSL of order 2, our results with 200, 1616 and 2000 obstacles show a definitive lead in performance for the direct family (Mumps, Lapack and Scalapack) compared with the GMRES family (with or without preconditioning). In particular, Lapack and its parallel version Scalapack lead in efficiency, with a great gap between them and the iterative family. While Mumps is less adapted because of the denseness of the multi-scattering linear systems, it still takes less time than the iterative family; the latter has difficulties converging for 1616 and 2000 obstacles. For 2000 obstacles and a visualization on a $800 \times 800$ grid, 330 Scalapack (on 16 cores) with Hermite interpolation (on 16 cores) takes 1 min 10 secs, c.f. Table 5 . 


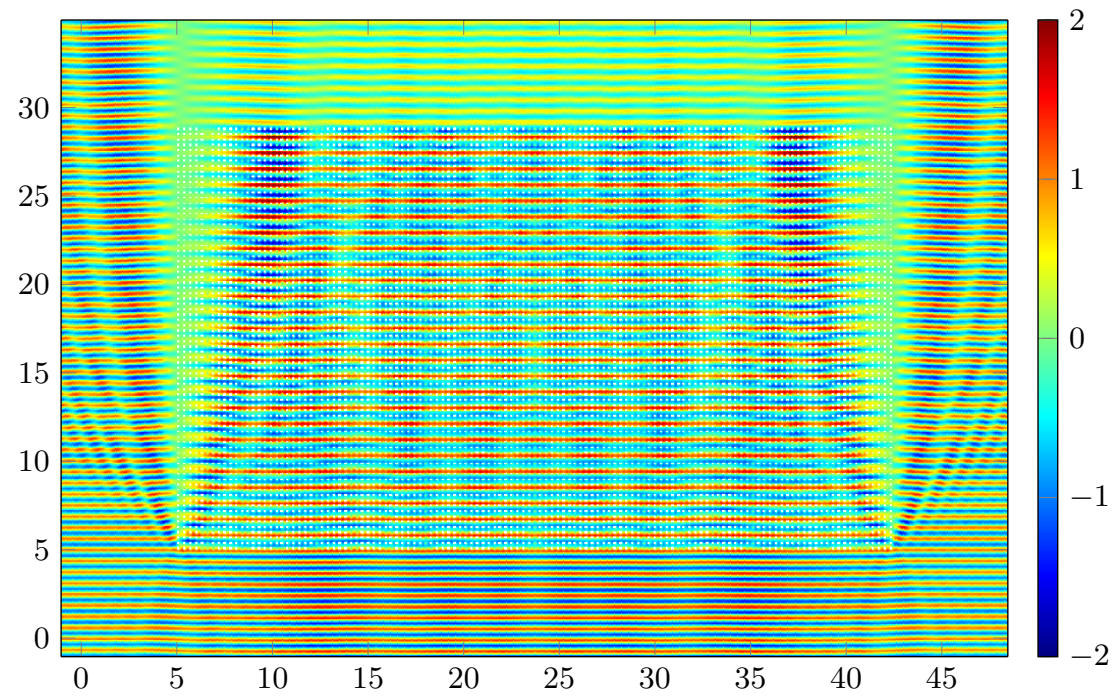

Figure 10: (Real part of) soft-scattered total wave of a PW of $90^{\circ}$ with $\lambda \sim 0.63$ by 10000 obstacles with $\mathrm{r}=0.03$ and $\mathrm{d}=0.3$. FSSL approx order $=2$. Matrix size $=$ $50000 \times 50000$. Resolution by Scalapack on 48 processors. Visualization is $800 \times 800$ grid on domain $79 \lambda \times 57 \lambda$. Total time $\sim 25$ mins. (Num. Exp. 6)

It is in the setting of far-apart obstacles, that we observe the advantage of the iterative solvers. We note the prominent robustness of the Lower-Upper Symmetric Gauss-Seidel (LUSGS) and Symmetric Gauss-Seidel (SGS) precon335 ditioners above the rest. These preconditioners are twice as fast as Lapack, and are almost comparable in performance to Scalapack. This robustness of iterative solvers was observed in [21, 6], however for their dense media. Thus we have arrived a different conclusion regarding the robustness of the direct and iterative families. In addition, among the family of preconditioners, we did not observe 340 the robustness of the Jacobi preconditioner, as noted in [21, 6]. As mentioned, it is the LUSGS and SGS that are very robust, being head-to-head with Scalapack on several processors for far-apart obstacles, and when the obstacles are close together, being the only preconditioners that converge. We also tested to see up to how many obstacles FSSL and its solvers can handle within a 'reasonable' 345 implementation time. For $10^{4}$ small and closely-spaced circular obstacles, the linear system is of size 50000 , and the only ${ }^{35}$ solvers that can handle this case is Scalapack with calculation time on 48 cores is $\sim 25$ mins, c.f. Exp. 6.

In $[8$, iterative solvers are also used to resolve the multiple scattering linear

\footnotetext{
${ }^{35}$ However, this might change with the parallelization and optimization of the codes for the iterative solvers with SGS and LUSGS solvers. Since the codes for the iterative solvers are currently sequential, with optimization and parallelization their performance can be improved (for far-apart obstacles).
} 
systems (associated with the modified layer Ansatz), and the (pre-processing) CPU time for circular (but larger, with radius $\lambda$ ) obstacles are substantially longer than what is observed for our experiments and in [6] for the same number of (smaller) obstacles. Our codes do not take advantage of the Toeplitz structure of the off-diagonal blocks as in [6, 9, a point which should be incorporated in future work. Our performance time for solver Lapack is comparable to that 355 reported in [9] (for closely-spaced obstacles).

\section{Conclusion}

Our numerical experiments have shown the robustness of FSSL method in solving the multiple scattering problem for small circular obstacles in large homogeneous media. Being mesh-free, it can handle efficiently a large number 360 of obstacles on an infinite homogeneous domain, while allowing the size of the obstacles to be very small compared to the incident wavelength. In addition, the linear systems generated by the method have simple definition, and thus enable easy coding and implementation.

For optimality, different formations of obstacles require different solver types. Direct Solvers (Lapack and Scalapack) are more efficient than iterative solvers in treating the cases where the obstacles are close together. On the other hand, iterative solvers are more preferable when the obstacles are far apart; in particular, GMRES methods with preconditioners LUSGS and SGS outperform Lapack and are head-to-head with parallel Scalapack. In the family of precon370 ditioners, we note the prominent robustness of LUSGS and SGS preconditioners, above the rest (especially in comparison to Jacobi). In both settings, due to parallelization, Scalapack takes the least time among the solvers.

However, the choice of an optimal solver also depends on the type of applications. In inverse problems where one needs the value of the diffracted field 375 at a line array of receivers, the evaluation cost is low, the weakness of FSSL (post-processing cost) is thus diminished. This makes FSSL more adapted than a FEM-based method. Regarding the choice of solvers within the FSSL, in addition to their robustness shown in the paper, the direct solvers (Lapack and Scalapack) are additionally ideal for the pragmatic reasons that they provide 380 higher precision and allow multi-rh $\$ 36$, and that the forward and adjoint problem use the same factorization 37. Since their performance does not vary as that of iterative ones with the distance of the obstacles, and Scalapack is faster than GMRES in either configuration, it is the ideal solver for our application.

\footnotetext{
36 In inverse problem, e.g. with Full waveform inversion, one has to solve many forward problems (see Footnote 20 in Section 4). With several acquisition data, e.g. when there are different types of sources (for the same wavenumber), each of these forward problems comes with multiple right hand sides. As a result, a solver with multi-rhs feature shortens the resolution time for each forward problem, and thus the overall implementation time for the inversion.

37 Unlike the iterative family, they can shorten the resolution time for the adjoint problem by reusing the factorization of the coefficient matrix, already obtained in the forward problem.
} 
Another appeal of this solver is that, with parallelization and shared memory architecture, it can handle a larger number of obstacles; an extreme example was shown for the case of $10^{4}$ small and closely spaced obstacles which can only be handled by FSSL with Scalapack (on 48 processors), for a total cost of 25 minutes.

Current and future problems. Due to their observed robustness, we are currently applying FSSL using direct solvers to reconstruct the positions of multiple defects in a homogeneous media by full-waveform inversion. A future work will treat the transmission problems for both acoustic-acoustic and acoustic-elastic interaction. A mathematical discussion for acoustic inclusions can be found in 28, 7. For extensions to general geometries, to maintain the robustness observed for disc geometry, we will need an efficient integration quadrature rule to numerically evaluate the components of the multiple-scattering linear systems 38 and handle the weak singularity of the Green kernel. This should be compared with other methods e.g. Nÿstrom, collocation, and in view of the results and more sophisticated methods in [11, 5, 29, 10, for a low number of 400 obstacles. In addition, future optimization of the codes should take into account the Toeplitz structure of the multiple-scattering matrix, and be bench-marked with the performance in 9 .

\section{Acknowledgement}

This work is part of a research program partially funded by Le Conseil Régional Nouvelle Aquitaine and the European Union's Horizon 2020 research and innovation program under the Marie Sklodowska-Curie grant agreement No 644602. The authors would like to thank MBarek Fares for providing us the original codes of GMRES. The authors would also like to thank the referee and reviewers for their constructive comments.

\section{Appendix A. Lemma for Fredholmness}

We will need the following uniqueness results (for the proof of Lemma 6).

Theorem 4 (Uniqueness). Hypothesis for the boundary : $\Gamma_{\text {obs }} \in \mathcal{C}^{2}$.

1. The EDP (5) and ENP have at most one solution.

2. The EIP (6) has at most one solution if $\operatorname{Im}(\bar{\kappa} \mathrm{i} \lambda) \geq 0$, on $\boldsymbol{\Gamma}_{\text {obs }}$.

\footnotetext{
${ }^{38}$ The RHS of $\sqrt{28}$ is a double line integral. For example, when $\alpha=D$,$$
\left(\mathbf{A}_{D, I J}\right)_{l k}=\int_{\Gamma_{I}} \mathbf{w}_{I, l} \int_{\Gamma_{J}} \overline{G_{\kappa}(x, y) \mathbf{w}_{J, k}} d s\left(\Gamma_{J}\right) d s\left(\Gamma_{I}\right)
$$$$
=\int_{0}^{2 \pi} \int_{0}^{2 \pi} e^{\mathrm{i} l \theta} \overline{G_{\kappa}\left(\gamma_{I}(\theta), \gamma_{J}(\tilde{\theta})\right)} e^{-\mathrm{i} k \tilde{\theta}}\left|\phi_{I}^{\prime}(\theta)\right|\left|\phi_{J}^{\prime}(\tilde{\theta})\right| d \tilde{\theta} d \theta .
$$ 
For the proof of EDP and ENP, we refer to [24, Thm 3.13]; for EIP, we refer to [24, Theorem 3.37]. For our investigation, we will only consider real and positive parameters, as a result, uniqueness is guaranteed.

The invertibility of the multiple-scattering linear system in Proposition 2 needs Lemmas 5 for Fredholmnes of $\mathbf{A}_{\alpha}$ and 6 for their injectivity. Their proofs 420 rely on the mapping properties of the surface operator, listed in $(8)$ and the following, for their proof see [22, Theorem 7.3]. For the following discussion, we drop the first index (denoting the interface) in their notation in (7) and write $\mathrm{S}_{\kappa}, \mathrm{D}_{\kappa}$, and $\mathrm{D}_{\kappa}^{\prime}$ for the surface potentials along a simple and closed curve $\Gamma$ at wavenumber $\kappa$.

1. When $\kappa=\mathrm{i}$ (with $\mathrm{i}^{2}=-1$ ), $\mathrm{S}_{\mathrm{i}}$ is $H^{-1 / 2}(\Gamma)$ - coercive, i.e.

$$
\left\langle\mathrm{S}_{\mathrm{i}} \phi, \phi\right\rangle_{H^{1 / 2}(\Gamma), H^{-1 / 2}(\Gamma)} \geq C\|\phi\|_{H^{-1 / 2}(\Gamma)}^{2} .
$$

2. If $\kappa^{2}$ is not a Dirichlet eigenvalue of $-\Delta$ in $\Omega$, then the SL potential at wavenumber $\kappa, \mathrm{S}_{\kappa}: H^{-1 / 2}(\Gamma) \rightarrow H^{1 / 2}(\Gamma)$ is an isomorphism with bounded inverse.

3. For $-1 \leq s \leq 1$, the following operators are compact and bounded

$$
\mathrm{S}_{\kappa}: H^{s-1 / 2}(\Gamma) \longrightarrow H^{s-1 / 2}(\Gamma), \mathrm{S}_{\kappa}-\mathrm{S}_{\mathrm{i}}: H^{-1 / 2}(\Gamma) \longrightarrow H^{1 / 2}(\Gamma),
$$

We will also use following properties of the jump of single-layer potentials,

$$
\llbracket \gamma_{0} \mathcal{S}_{\kappa} \psi \rrbracket=0 \text { in } H^{1 / 2}(\Gamma) \quad, \quad \llbracket \gamma_{1} \mathcal{S}_{\kappa} \psi \rrbracket=-\psi \text { in } H^{-1 / 2}(\Gamma) .
$$

The sign convention on the jump is

$$
\llbracket \gamma_{0} f \rrbracket:=\gamma_{0}^{+} f-\gamma_{0}^{-} f ; \llbracket \gamma_{1} f \rrbracket:=\gamma_{1}^{+} f-\gamma_{1}^{-} f=\llbracket \nabla f \rrbracket \cdot n .
$$

Lemma 5. For $\alpha=D$, Im and $N$, the operator $\mathbf{A}_{\alpha}$ is Fredholm.

Proof. For each type of boundary conditions, we will show that $\mathbf{A}_{\alpha}$ can be written as a product of an invertible operator and a compact perturbation of the identity map. We recall the notation that $S_{I, \mathrm{i}}$ is the single-layer potential, along boundary $\Gamma_{I}$ of obstacle $I$, at wavenumber i.

Dirichlet: Decompose $\mathbf{A}_{D}$ as follows,

$$
\mathbf{A}_{D}=\mathbf{B}+\mathbf{C}_{D} \quad, \quad \mathbf{B}:=\operatorname{diag}\left(\mathrm{S}_{1, \mathrm{i}}, \mathrm{S}_{2, \mathrm{i}}, \ldots, \mathrm{S}_{N, \mathrm{i}}\right) .
$$

Here $\mathbf{B}$ is zero except along its diagonal block. Since its components $S_{I, \mathrm{i}}$ : $H^{-1 / 2}\left(\Gamma_{I}\right) \rightarrow H^{1 / 2}\left(\Gamma_{I}\right)$ are invertible with bounded inverse $\mathbf{S}_{I, \mathrm{i}}^{-1}$, operator $\mathbf{B}$ is invertible with bounded inverse

$$
\mathbf{B}^{-1}=\operatorname{diag}\left(\mathrm{S}_{1, \mathrm{i}}^{-1}, \mathrm{~S}_{2, \mathrm{i}}^{-1}, \ldots, \mathrm{S}_{N, \mathrm{i}}^{-1}\right): \mathbb{H}_{1 / 2}\left(\boldsymbol{\Gamma}_{\text {Obs }}\right) \longrightarrow \mathbb{H}_{-1 / 2}\left(\boldsymbol{\Gamma}_{\text {Obs }}\right) .
$$

We next consider the remainder $\mathbf{C}$. Its components are given by

$$
\mathbf{C}_{I J}=\mathrm{S}_{I J, \kappa}, I \neq J ; \quad \mathbf{C}_{I J}=\mathrm{S}_{I, \kappa}-\mathrm{S}_{I, \mathrm{i}}, I=J .
$$


Since $\mathrm{S}_{I J, \kappa}: H^{-1 / 2}\left(\Gamma_{J}\right) \rightarrow H^{1 / 2}\left(\Gamma_{I}\right)$, with $I \neq J$, and $\mathrm{S}_{I, \kappa}-\mathrm{S}_{I, \mathrm{i}}: H^{-1 / 2}\left(\Gamma_{I}\right) \rightarrow$ $H^{1 / 2}\left(\Gamma_{I}\right)$ are bounded and compact, so is $\mathbf{C}_{I J}: H^{-1 / 2}\left(\Gamma_{J}\right) \rightarrow H^{1 / 2}\left(\Gamma_{I}\right)$, and

$\mathbf{K}:=\mathbf{B}^{-1} \mathbf{C}: \mathbb{H}_{-1 / 2}\left(\boldsymbol{\Gamma}_{\text {Obs }}\right) \longrightarrow \mathbb{H}_{-1 / 2}\left(\boldsymbol{\Gamma}_{\text {Obs }}\right)$ is bounded and compact

As a result, $\mathbf{A}_{D}$ is a Fredholm operator with

$$
\left(\mathbf{A}_{D}\right)_{\mathbb{H}_{-1 / 2} \rightarrow \mathbb{H}_{1 / 2}}=\mathbf{B}_{\mathbb{H}_{-1 / 2} \rightarrow \mathbb{H}_{1 / 2}}\left(\mathbf{I d}_{\mathbb{H}_{-1 / 2} \rightarrow \mathbb{H}_{-1 / 2}}+\mathbf{K}_{\mathbb{H}_{-1 / 2} \rightarrow \mathbb{H}_{-1 / 2}}\right) \text {. }
$$

Impedance and Neumann: For $\alpha=I$ and $N$, we can readily decompose

$$
\mathbf{A}_{\alpha}=-\frac{1}{2}\left(\mathbf{I d}-\mathbf{K}_{\alpha}\right) \quad, \quad \mathbf{K}_{I J}=2 \mathrm{D}_{I J, \kappa}^{\prime}+2 \mathrm{i} \lambda \mathrm{S}_{I J, \kappa} .
$$

Since $\mathrm{S}_{I J, \kappa}, \mathrm{D}_{I J, \kappa}^{\prime}: H^{-1 / 2}\left(\Gamma_{J}\right) \rightarrow H^{-1 / 2}\left(\Gamma_{I}\right)$ is bounded and compact,

$$
\mathbf{K}_{\alpha}: \mathbb{H}_{-1 / 2}\left(\boldsymbol{\Gamma}_{\text {Obs }}\right) \longrightarrow \mathbb{H}_{-1 / 2}\left(\boldsymbol{\Gamma}_{\text {Obs }}\right) \text { is bounded and compact. }
$$

Lemma 6. If $\kappa^{2}$ is not a Dirichlet eigenvalue for $-\Delta$ inside domain $\Omega_{I}$ with $1 \leq 1 \leq N$, then $\mathbf{A}_{\alpha}$ is injective, with $\alpha=D, N$, Im.

Proof. We will make extensive use of the well-posedness of the exterior boundary values problems listed in Theorem 4 . For $\psi_{I} \in H^{-1 / 2}\left(\Gamma_{I}\right)$ such that

$$
\mathbf{A}_{\alpha} \boldsymbol{\psi}=0 \quad, \quad \boldsymbol{\psi}=\left(\psi_{1}, \ldots, \psi_{N}\right),
$$

we want to show that $\boldsymbol{\psi}=0$. This would follow, if $u$ defined as

$$
u:=\mathcal{S}_{1, \kappa} \psi_{1}+\ldots+\mathcal{S}_{2, \kappa} \psi_{N},
$$

is zero in $\Omega_{\text {ext }}$. This can be seen as follows. Since $\mathcal{S}_{I}$ extends to all $\mathbb{R}^{2}, u$ as defined solves $\left(-\Delta-\kappa^{2}\right) u=0$ both in $\Omega_{\text {ext }}$ and $\Omega_{I}, 1 \leq I \leq N$. Under the assumption that $u=0$ in $\Omega_{\text {ext }}$, we have $\gamma_{0, I}^{+} u=0,1 \leq I \leq N$. By the continuity of zero-th order trace of the single layers, we have $\gamma_{0, I}^{-} u=\gamma_{0, I}^{+} u=$ 0 . This means that $u$ solves the interior Dirichlet problem at wavenumber $\kappa$ with zero boundary value in each domain $\Omega_{I}$. By assumption, $\kappa^{2}$ is not a Dirichlet eigenvalue on $\Omega_{I}$, this forces $u=0$ in $\Omega_{I}$. As a result, $\gamma_{1, I}^{-} u=0,1 \leq I \leq N$. On the other hand, under the current assumption $u=0$ in $\Omega_{\text {ext }}$, we have

$$
\llbracket \gamma_{1, I} u \rrbracket=0 \quad, \quad I=1, \ldots, N .
$$

Now, we can use the jump identity for single layer, $\llbracket \gamma_{1, I} \mathcal{S}_{I, \kappa} \psi_{I} \rrbracket=\psi_{I}$ and $\llbracket \gamma_{1, I} \mathcal{S}_{J, \kappa} \psi_{J} \rrbracket=0, I \neq J$, c.f. $[9]$, to obtain

$$
\llbracket \gamma_{1, I} u \rrbracket=\llbracket \gamma_{1, I} \mathcal{S}_{1} \psi_{1} \rrbracket+\ldots+\llbracket \gamma_{1, I} \mathcal{S}_{2} \psi_{N} \rrbracket=\psi_{I} .
$$

As as result, we have shown that, if $u$ defined in $($ A.6 $)$ is zero in $\Omega_{\text {ext }}$, and under non-Dirichlet eigenvalue assumption on $\kappa^{2}$, then the jump $\psi_{I}=\llbracket \gamma_{1, I} u \rrbracket=0$ and thus $\boldsymbol{\psi}=0$. 
Now it remains to prove that this assumption on $\kappa^{2}, u$ as defined in A.6 $u=0$ for $\Omega_{\text {ext }}$.

450

\section{Appendix B. Hermite cubic interpolation for Hankel functions}

In evaluating the diffracted field 46 for post-processing in FSSL, the most expensive 39 operation is the computation of Hankel functions at distances between each evaluation point and each obstacle. In particular, consider $N$ obstacles and a domain of interest $[a, b] \times[c, d]$ meshed by a $M \times M^{\prime}$ structured grid. For every $x$ on this grid, $1 \leq I \leq N$ and $0 \leq k \leq \mathbf{m}$, we need to calculate

$$
\mathrm{H}_{k}^{(1)}\left(\kappa\left\|x-\mathbf{x}_{I}\right\|\right) \text {. }
$$

If we ignore ${ }^{40}$ the cost of several orders $k$, the cost is

$$
\text { Total cost } \sim\left(\begin{array}{c}
\text { Cost for }{ }_{\text {of }}^{\text {B.1 }} \\
\text { eval }
\end{array}\right) \times M \times M^{\prime} \times N .
$$

Following is a discussion of the method we chose in this paper to reduce the cost associated with the factor $M \times M^{\prime}$. Denote by $\Omega_{\text {visu }}$ the set of points of the visualization grid which are outside the obstacles.

In our codes, we use the intrinsic functions Bessel_JN and Bessel_YN of Fortran90 42 , The recurrence relation and identity, c.f. [26, Chapter 9 p.361], followed by Bessel functions of integer orders, are also used

$$
\begin{aligned}
\mathrm{Z}_{k-1}(z)+\mathrm{Z}_{k+1}(z) & =\frac{2 k}{z} \mathrm{Z}_{k}(z) \\
\mathrm{Z}_{-k}(z) & =(-1)^{k} \mathrm{Z}_{k}(z) .
\end{aligned}
$$

\footnotetext{
39 Remark 9 shows that the cost of computing exp(i.) and the Hankel functions are much more expensive than standard operations (multiplication, addition). For the current codes, we only focus on reducing the cost of Hankel functions, since this one is about twice the cost of the exponential.

40 Higher order Hankels are calculated by recurrence relations, thus the cost due to the order of approximation (generally $<4$ ) is less substantial than other factors.

${ }^{41}$ This is just one of the many ways to reduce the cost.

${ }^{42}$ Their description can be found at https://gcc.gnu.org/onlinedocs/gfortran/BESSEL_ $005 f$ JN.html and https://gcc.gnu.org/onlinedocs/gfortran/BESSEL_005fYN.html. These two functions allow vectorization in one of the input variables, either the order or the point of evaluation. For the exact post-processing, we take advantage of the first option, while for the interpolation, we use the latter.
} 
In the above expressions, $\mathrm{Z}=\mathrm{J}$ (the Bessel function of the first kind) or $\mathrm{Y}$ (Bessel function of the second kind). In the following discussion, the current order of approximation is $\mathbf{m}$.

Step 1. We create a grid on which the exact value of the Hankel and its derivatives for order $k$ with $0 \leq k \leq \mathbf{m}$ are calculated for each node.

- Estimate a lower bound $r_{\min }$ and an upper bound $r_{\max }$ of the set

$$
\left\{\left\|x-\mathbf{x}_{I}\right\| \mid 1 \leq I \leq N, x \in \Omega_{\text {visu }}\right\} .
$$

This is the set of possible distances between any point in $\Omega_{\mathrm{visu}}$ to the center of any of the obstacles.

- For our experiments, we use interpolation step size ${ }^{43} h_{\text {inter }}=0.1$. Create a vector $V_{\text {inter }}$ with endpoints $\kappa \times \mathrm{r}_{\min }$ and $\kappa \times \mathrm{r}_{\max }$ and increment $h_{\text {inter }}$, i.e. $V_{\text {inter }}=\left[\kappa \times \mathrm{r}_{\min }: h_{\text {inter }}: \kappa \times \mathrm{r}_{\max }\right]$. This vector is of length

$$
L=\left[\frac{\kappa \times \mathrm{r}_{\max }-\kappa \times \mathrm{r}_{\min }}{h_{\text {inter }}}\right]+1,[\cdot] \text { is the floor function } .
$$

Also denote by $t_{i}$ the components of $V_{\text {inter }}$, i.e. $t_{i}=\kappa \times \mathrm{r}_{\min }+i \times h_{\text {inter }}$.

- We call Bessel_YN $\left(0, V_{\text {inter }}\right)$ and Bessel_YN $\left(1, V_{\text {inter }}\right)$ to obtain the values of $\mathrm{Y}_{0}$ and $\mathrm{Y}_{1}$ at each node of vector $V_{\text {inter }}$. Higher orders $2 \leq k \leq \mathbf{m}+1$ are calculated by using the recurrence relation (B.3) 'upwardly'. Order $\mathbf{m}+1$ is used for calculating derivatives of the Bessel functions in the next step. The calculation for Bessel J is carried similarly but 'downward' ${ }^{44}$.

- The Hankel function of the first kind $\mathrm{H}_{n}^{(1)}$ and its derivatives $\mathrm{H}_{n}^{(1) \prime}$ on each node of $V_{\text {inter }}$ and for orders $0 \leq k \leq \mathbf{m}$ are obtained by the identities, c.f. [26. Chapter 9 p.361]

$$
\mathrm{H}_{k}^{(1)}=\mathrm{J}_{k}+\mathrm{i} \mathrm{Y}_{k} \quad, \quad 2 \mathrm{H}_{k}^{(1) \prime}=\mathrm{H}_{k-1}^{(1)}-\mathrm{H}_{k+1}^{(1)} \quad, \quad \mathrm{H}_{0}^{(1) \prime}=-\mathrm{J}_{1}-\mathrm{i} \mathrm{Y}_{1} .
$$

Step 2. After having obtained the value $\mathrm{H}_{k}^{(1)}$ and its derivative for each node point $t_{i}$ of $V_{\text {inter }}$, we next calculate the function $\mathfrak{p}_{k}$ which gives the interpolating value of $\mathrm{H}_{k}^{(1)}$ at any point $t \in\left[t_{1}, t_{L}\right]$. We use Hermite cubic interpolation,

\footnotetext{
${ }^{43}$ This step size corresponds to a precision of $10^{-5}$ in our numerical experiments. This is the relative difference in (discrete) $L^{2}$ norm between the result obtained by exact evaluation and interpolated one.

${ }^{44}$ We call Bessel_JN $\left(\mathbf{m}+1, V_{\text {inter }}\right)$ and $\operatorname{Bessel}_{-} \mathrm{JN}\left(\mathbf{m}, V_{\text {inter }}\right)$. Orders $k$ with $0 \leq k \leq$ $\mathbf{m}-1$ are calculated using the recurrence relation (B.3) in the downward direction. The reason for choosing 'downward' or 'upward' is due to numerical stability. For $\mathrm{Y}_{k}$ the upward recurrence B.3 is stable, while for $J_{k}$ it is the downward direction that is stable, c.f. 30 p. 301]
} 
and follow the discussion in [31, p. 48]. The interpolating function $\mathfrak{p}_{k}$ on each interval $\left[t_{i}, t_{i+1}\right]$ is equal to the cubic polynomial $\mathfrak{p}_{k, i}$ defined by the properties

$$
\begin{gathered}
\mathfrak{p}_{k, i}\left(t_{i}\right)=\mathrm{H}_{k}^{(1)}\left(t_{i}\right) \quad, \quad \mathfrak{p}_{k, i}\left(t_{i+1}\right)=\mathrm{H}_{k}^{(1)}\left(t_{i+1}\right) \\
\mathfrak{p}_{k, i}^{\prime}\left(t_{i}\right)=\mathrm{H}_{k}^{(1) \prime}\left(t_{i}\right) \quad, \quad \mathfrak{p}_{k, i}^{\prime}\left(t_{i+1}\right)=\mathrm{H}_{k}^{(1) \prime}\left(t_{i+1}\right)
\end{gathered}
$$

We write $\mathfrak{p}_{k, i}$ (which is also $\left.\left.\mathfrak{p}_{k}\right|_{\left[t_{i}, t_{i+1}\right]}\right)$ as

$\mathfrak{p}_{k, i}(t)=a_{k, i ; 0}+a_{k, i ; 1}\left(t-t_{i}\right)+\left(t-t_{i}\right)\left(t-t_{i+1}\right)\left[a_{k, i ; 2}\left(t-t_{i}\right)+a_{k, i ; 3}\left(t-t_{i+1}\right)\right]$,

and calculate its coefficients $a_{k, i ; 0}, \ldots, a_{k, i ; 3}$ by formula (10.10) in [31, p. 48].

Step 3. For each $k$ with $0 \leq k \leq \mathbf{m}, I$ with $1 \leq I \leq N$, and each point $x$ on the $M \times M^{\prime}$ structured grid, we calculate $\mathrm{H}_{k}^{(1)}\left(\kappa\left\|x-\mathbf{x}_{I}\right\|\right)$ as follows. We first determine the interval in which $\kappa\left\|x-\mathbf{x}_{I}\right\|$ belongs; in particular,

$$
\kappa\left\|x-\mathbf{x}_{I}\right\| \in\left[t_{i}, t_{i+1}\right] \quad \text { with } \quad i=\left[\frac{\kappa\left\|x-\mathbf{x}_{I}\right\|-t_{1}}{h_{\text {inter }}}\right]+1 \text {. }
$$

Since $\mathrm{H}_{k}^{(1)}$ is approximated by the interpolating polynomial $\mathfrak{p}_{k, i}$ on this interval, we use

$$
\mathrm{H}_{k}^{(1)}\left(\kappa\left\|x-\mathbf{x}_{I}\right\|\right) \sim \mathfrak{p}_{k, i}\left(\kappa\left\|x-\mathbf{x}_{I}\right\|\right) .
$$

The negative orders $-\mathbf{m} \leq k<0$ are calculated using Identity (B.4).

470 Remark 8. In practice, to speed up the implementation, Step 1-2 is 'vectorized', and Step 3 is parallelized.

Remark 9 (CPU time of some instrinsic functions in Fortran90). For a real vector $\mathbf{z}$ of length $2 \times 10^{7}$ whose components are randomly chosen, we list $C P U$ time for the following intrinsic operations computed by Fortran90 with compiler mpi90,

\begin{tabular}{cc|cc|cc} 
Operation & Time $(s)$ & Operation & Time $(s)$ & Operation & Time $(s)$ \\
\hline $\mathbf{z}+\mathbf{z}$ & 0.03 & $\cos (\mathbf{z})$ & 1.95 & $\mathrm{~J}_{0}(\mathbf{z})$ & 3.51 \\
$\mathbf{z}^{2}$ & 0.15 & $\sin (\mathbf{z})$ & 1.96 & $\mathrm{~J}_{1}(\mathbf{z})$ & 3.55 \\
$\exp (\mathbf{z})$ & 1.63 & $\cos (\mathbf{z})+\mathrm{i} \sin (\mathbf{z})$ & 3.80 & $\mathrm{Y}_{0}(\mathbf{z})$ & 3.55 \\
$\exp (\mathrm{i} \mathbf{z})$ & 1.14 & & & $\mathrm{Y}_{0}(\mathbf{z})$ & 3.63
\end{tabular}

Time costs for calculating $\mathrm{Y}_{k}(\mathbf{z})$ and $\mathrm{J}_{k}(\mathbf{z})$ for all (integer) orders $k$ between 0 and 4, using upward recurrence (B.3), (described in Step 1c), are $8.01 \mathrm{~s}$ and $7.91 \mathrm{~s}$ respectively. These two costs give the overall one for $\mathrm{H}_{k}^{(1)}(\mathbf{z})$ (obtained 475 by adding the cost of Bessel $\mathrm{J}_{k}$ and that of Bessel $\mathrm{Y}_{k}$ ) which is $16.30 \mathrm{~s}$.

\section{Bibliography}

[1] D. Givoli, Numerical methods for problems in infinite domains, Vol. 33, Elsevier, 2013. 
[2] F. Nataf, Absorbing boundary conditions and perfectly matched layers in wave propagation problems (2013).

[3] F.-J. Sayas, The validity of Johnson-Nédélec's BEM-FEM coupling on polygonal interfaces, SIAM Journal on Numerical Analysis 47 (5) (2009) 3451-3463.

[4] A. Kirsch, P. Monk, An analysis of the coupling of finite-element and Nyström methods in acoustic scattering, IMA Journal of numerical analysis 14 (4) (1994) 523-544.

[5] M. Ganesh, C. Morgenstern, High-order FEM-BEM computer models for wave propagation in unbounded and heterogeneous media: Application to time-harmonic acoustic horn problem, Journal of Computational and Applied Mathematics 307 (2016) 183-203.

[6] X. Antoine, K. Ramdani, B. Thierry, Wide frequency band numerical approaches for multiple scattering problems by disks, Journal of Algorithms \& Computational Technology 6 (2) (2012) 241-259.

[7] B. Thierry, Analyse et simulations numériques du retournement temporel et de la diffraction multiple, Ph.D. thesis, Université Henri Poincaré-Nancy I (2011).

[8] M. Ganesh, S. C. Hawkins, An efficient algorithm for simulating scattering by a large number of two dimensional particles, ANZIAM Journal 52 (2011) $139-155$.

[9] F. A. Amirkulova, A. N. Norris, Acoustic multiple scattering using recursive algorithms, Journal of Computational Physics 299 (2015) 787-803.

[10] S. Falletta, G. Monegato, Exact nonreflecting boundary conditions for exterior wave equation problems, Publications de l'Institut Mathématique 96 (110) (2014) 103-123.

[11] M. Ganesh, I. Graham, A high-order algorithm for obstacle scattering in three dimensions, Journal of Computational Physics 198 (1) (2004) 211242 .

[12] A. Bendali, P.-H. Cocquet, S. Tordeux, Approximation by multipoles of the multiple acoustic scattering by small obstacles in three dimensions and application to the foldy theory of isotropic scattering, Archive for Rational Mechanics \& Analysis 219 (3) (2016).

[13] D. P. Challa, M. Sini, On the justification of the Foldy-Lax approximation for the acoustic scattering by small rigid bodies of arbitrary shapes, Multiscale Modeling \& Simulation 12 (1) (2014) 55-108.

[14] P. Martin, Multiple Scattering: Interaction of Time-Harmonic Waves with N Obstacles, Cambridge University Press, 2006. 
[15] P. Martin, Corrections and additions.

[16] R. Kress, Boundary integral equations in time-harmonic acoustic scattering, Mathematical and Computer Modelling 15 (3) (1991) 229-243.

[17] X. Antoine, B. Thierry, Spectral and condition number estimates of the acoustic single-layer operator for low-frequency multiple scattering in dilute media, Computer Methods in Applied Mechanics and Engineering 265 (2013) 242-256.

[18] B. Thierry, X. Antoine, Spectral and condition number estimates of the acoustic single-layer operator for low-frequency multiple scattering in dense media, Journal of Computational and Applied Mathematics 239 (2013) 380-395.

[19] B. Thierry, X. Antoine, C. Chniti, H. Alzubaidi, $\mu$-diff: an open-source Matlab toolbox for computing multiple scattering problems by disks, Computer Physics Communications 192 (2015) 348-362.

[20] V. Frayssé, L. Giraud, S. Gratton, J. Langou, A set of GMRES routines for real and complex arithmetics on high performance computers, Tech. rep., CERFACS, tR/PA/03/3 (1997).

[21] X. Antoine, K. Ramdani, B. Thierry, Étude numérique de la résolution par équations intégrales de la diffraction multiple par des disques, in: 10ème Congrès Français d'Acoustique, 2010.

[22] F. Cakoni, D. Colton, A Qualitative Approach to Inverse Scattering Theory, Springer, 2014.

[23] F. Hettlich, Fréchet derivatives in inverse obstacle scattering, Inverse problems 11 (2) (1995) 371.

[24] D. Colton, R. Kress, Integral Equation Methods in Scattering Theory, SIAM, Society for Industrial and Applied Mathematics, Philadelphia, 2013.

[25] R. Kress, Linear Integral Equation, 3rd Edition, Springer, 2014.

[26] M. Abramowitz, I. A. Stegun, Handbook of mathematical functions: with formulas, graphs, and mathematical tables, Vol. 55, Courier Corporation, 1964 .

[27] S. Sauter, C. Schwab, Boundary Element Methods, Springer, 2011.

[28] H. Barucq, J. Chabassier, H. Pham, S. Tordeux, A study of the numerical robustness of single-layer method with Fourier basis for multiple obstacle scattering in homogeneous media, Research Report RR-8988, Inria Bordeaux Sud-Ouest (Dec. 2016).

[29] M. Ganesh, S. C. Hawkins, Simulation of acoustic scattering by multiple obstacles in three dimensions, ANZIAM Journal 50 (2008) 31-45. 
[30] W. H. Press, Numerical recipes: The art of scientific computing, Cambridge university press, 2007.

[31] E. Hairer, W. Gerhard, Introduction à l'Analyse Numérique, Université de Genève - Section de mathématiques, 2005.

URL https://archive-ouverte.unige.ch/unige:12656 